\title{
Observation and Interpretation of Motional Sideband Asymmetry in a Quantum Electromechanical Device
}

\author{
A. J. Weinstein, ${ }^{1,2}$ C. U. Lei, ${ }^{1,2}$ E. E. Wollman, ${ }^{1,2}$ J. Suh, ${ }^{1,2, \dagger}$ A. Metelmann, ${ }^{3}$ A. A. Clerk, ${ }^{3}$ and K. C. Schwab ${ }^{1,2, *}$ \\ ${ }^{1}$ Applied Physics, Caltech, Pasadena, California 91125, USA \\ ${ }^{2}$ Kavli Nanoscience Institute, Caltech, Pasadena, California 91125, USA \\ ${ }^{3}$ Department of Physics, McGill University, Montreal, Quebec H3A 2T8, Canada
}

(Received 15 May 2014; revised manuscript received 31 July 2014; published 7 October 2014)

\begin{abstract}
Quantum electromechanical systems offer a unique opportunity to probe quantum noise properties in macroscopic devices, properties that ultimately stem from Heisenberg's uncertainty relations. A simple example of this behavior is expected to occur in a microwave parametric transducer, where mechanical motion generates motional sidebands corresponding to the up-and-down frequency conversion of microwave photons. Because of quantum vacuum noise, the rates of these processes are expected to be unequal. We measure this fundamental imbalance in a microwave transducer coupled to a radiofrequency mechanical mode, cooled near the ground state of motion. We also discuss the subtle origin of this imbalance: depending on the measurement scheme, the imbalance is most naturally attributed to the quantum fluctuations of either the mechanical mode or of the electromagnetic field.
\end{abstract}

DOI: 10.1103/PhysRevX.4.041003

\section{INTRODUCTION}

A fascinating aspect of quantum measurement is that the outcome of experiments and the apparent nature of the object under study depend critically on the properties of both the system and the measurement scheme [1]. An excellent illustration is found when considering measurements of the quantum harmonic oscillator. When measured with an ideal energy detector, the observed signals will demonstrate energy-level quantization [2,3]; however, if measured instead with an ideal position detector, no evidence of quantized energy levels is found and the measured signals appear to be those of a very cold, classical oscillator [4,5]. The details of the measurement are as essential to the apparent nature of the system under study as the properties of the system itself - succinctly expressed by Roy Glauber, "A photon is what a photodetector detects" [6].

To describe the measured noise of quantum systems, it is often useful to make use of so-called quantum noise spectral densities, which, in general, are not symmetric functions of frequency: $S_{x x}(-\omega) \neq S_{x x}(+\omega)$, where $S_{x x}(\omega)$ is the spectral density of the observable $x(t)$, defined as the Fourier transform of $\langle\hat{x}(t) \hat{x}(0)\rangle$ [7]. For a quantum harmonic oscillator, the negative- and positive-frequency sides

*To whom all correspondence should be addressed. schwab@caltech.edu

${ }^{\dagger}$ Present address: Korea Research Institute of Standards and Science, Daejeon 305-340, Republic of Korea.

Published by the American Physical Society under the terms of the Creative Commons Attribution 3.0 License. Further distribution of this work must maintain attribution to the author(s) and the published article's title, journal citation, and DOI.
Subject Areas: Mechanics, Quantum Physics, Quantum Information of this spectral density describe the ability of the system to emit or absorb energy. In the ground state, there is no ability for the harmonic oscillator to emit energy, so that $S_{x x}\left(-\omega_{m}\right)=0$. It can, however, absorb energy, and as a result, $S_{x x}\left(+\omega_{m}\right)=\left(4 / \gamma_{m}\right) x_{\mathrm{ZP}}^{2}$, where $x_{\mathrm{ZP}}=\sqrt{\hbar / 2 m \omega_{m}}$ is the amplitude of zero-point fluctuations for a mechanical oscillator with mass $m$, resonance frequency $\omega_{m}$, and damping rate $\gamma_{m}$. More generally, for a mechanical oscillator in a thermal state with an occupation factor $\bar{n}_{m}$, the spectral densities follow $S_{x x}\left(-\omega_{m}\right)=\left(4 / \gamma_{m}\right) x_{\mathrm{ZP}}^{2} \bar{n}_{m}$ and $S_{x x}\left(+\omega_{m}\right)=\left(4 / \gamma_{m}\right) x_{\mathrm{ZP}}^{2}\left(\bar{n}_{m}+1\right)$ [3]. This asymmetricin-frequency motional noise spectrum was first measured in atomic systems prepared in quantum ground states of motion [8-10], where the motional sideband absorption and fluorescence spectra were detected via photodetection.

Analogous quantum noise effects can also be studied in macroscopic mechanical systems, using electromechanical and optomechanical devices prepared and probed at quantum limits [5,11-13]. These systems exhibit the Ramanlike processes of the up- and down-conversion of photons, resulting from the parametric coupling between mechanical motion and electromagnetic modes of a resonant cavity; the rates of these processes should naturally mirror the asymmetry in the mechanical quantum noise spectral density $S_{x x}\left( \pm \omega_{m}\right)$. Recent experiments in optomechanics have demonstrated this expected imbalance between up- and down-converted sidebands $[14,15]$. Here, we demonstrate the analogous physics in a quantum circuit, where it is now microwave photons (not optical photons) that probe the mechanical motion.

We also address a subtlety about these measurements that originates from their use of linear detection of the scattered electromagnetic field: they measure the field 
amplitude (e.g., via heterodyne detection). This scheme is in contrast to measurements employing direct photodetection, where one filters the output light and counts photons associated with a motional sideband. Although the predicted and measured motional sideband asymmetries obtained using either detection method are identical [14-16], the interpretation is more nuanced when one employs linear field detection. As discussed by Khalili et al. [16], the asymmetry in this case can be fully attributed to the detector, namely, the presence of a precisely tuned correlation between the backaction noise generated by the measurement device and its imprecision noise (see Appendix B). We provide a simple exposition of this physics using standard input-output theory, which lets us easily track the scattering of incident vacuum fluctuations. In the case of linear detection of the cavity output field, the imbalance is naturally attributed to the input electromagnetic field fluctuations (classical and quantum); the intrinsic quantum fluctuations of the mechanical mode contribute equally to the up- and down-converted spectra. In contrast, in experiments that employ direct photodetection, the asymmetric quantum noise of the mechanical motion directly contributes to the imbalance of the output spectrum (in addition to any contribution from excess classical fluctuations in the input electromagnetic fields). After a brief discussion of these theoretical issues, we present measurements of the imbalance in a microwave-frequency electromechanical device.

\section{THEORY}

We begin with the Hamiltonian of our electromechanical system

$$
\hat{\mathcal{H}}=\hbar \omega_{c} \hat{a}^{\dagger} \hat{a}+\hbar \omega_{m} \hat{b}^{\dagger} \hat{b}+\hbar g_{0} \hat{a}^{\dagger} \hat{a}\left(\hat{b}+\hat{b}^{\dagger}\right),
$$

where $\hat{a}\left(\hat{a}^{\dagger}\right)$ is the annihilation (creation) operator of the microwave resonator mode with frequency $\omega_{c}, \hat{b}\left(\hat{b}^{\dagger}\right)$ is the annihilation (creation) operator of the mechanical resonator with frequency $\omega_{m}$, and $g_{0}$ is the parametric coupling strength between the two modes.

We consider the standard regime of a cavity strongly driven at frequency $\omega_{p}$, where dissipation is treated as per standard input-output theory [17]; we also consider a twosided cavity, which corresponds to our experimental setup. Writing the cavity and mechanical fields in terms of their classical and quantum parts $\hat{a}=e^{-i \omega_{p} t}(\bar{a}+\hat{d})$ and $\hat{b}=\bar{b}+\hat{c}$, we linearize to obtain the following Heisenberg-Langevin equations:

$$
\begin{aligned}
& \dot{\hat{d}}=-\left(i \Delta+\frac{\kappa}{2}\right) \hat{d}-i G\left(\hat{c}+\hat{c}^{\dagger}\right)-\sum_{\sigma=R, L} \sqrt{\kappa_{\sigma}} \hat{d}_{\sigma, \mathrm{in}}, \\
& \dot{\hat{c}}=-\left(i \omega_{m}+\frac{\gamma_{m}}{2}\right) \hat{c}-i G\left(\hat{d}+\hat{d}^{\dagger}\right)-\sqrt{\gamma_{m}} \hat{c}_{\mathrm{in}},
\end{aligned}
$$

where $\Delta=\omega_{c}-\omega_{p}, G=g_{0}|\bar{a}|$, and $\kappa=\kappa_{L}+\kappa_{R}\left(\gamma_{m}\right)$ is the microwave- (mechanical-) resonator damping rate. The operators $\hat{d}_{\sigma, \text { in }}(t)$ and $\hat{c}_{\text {in }}(t)$ describe noise incident on the microwave and mechanical resonator, respectively, and satisfy

$$
\begin{aligned}
\left\langle\hat{d}_{\sigma, \text { in }}(t) \hat{d}_{\sigma^{\prime}, \text { in }}^{\dagger}\left(t^{\prime}\right)\right\rangle & =\left(n_{\sigma}^{\text {th }}+\alpha\right) \delta_{\sigma, \sigma^{\prime}} \delta\left(t-t^{\prime}\right), \\
\left\langle\hat{d}_{\sigma, \text { in }}^{\dagger}(t) \hat{d}_{\sigma^{\prime}, \text { in }}\left(t^{\prime}\right)\right\rangle & =n_{\sigma}^{\text {th }} \delta_{\sigma, \sigma^{\prime}} \delta\left(t-t^{\prime}\right), \\
\left\langle\hat{c}_{\text {in }}(t) \hat{c}_{\text {in }}^{\dagger}\left(t^{\prime}\right)\right\rangle & =\left(n_{m}^{\text {th }}+\beta\right) \delta\left(t-t^{\prime}\right), \\
\left\langle\hat{c}_{\text {in }}^{\dagger}(t) \hat{c}_{\text {in }}\left(t^{\prime}\right)\right\rangle & =n_{m}^{\text {th }} \delta\left(t-t^{\prime}\right) .
\end{aligned}
$$

Here, $n_{m}^{\text {th }}\left(n_{\sigma}^{\text {th }}\right)$ denotes the amount of thermal fluctuations incident on the mechanical resonator (microwave resonator from port $\sigma$ ), and $\alpha$ and $\beta$ describe the quantum vacuum fluctuations driving the microwave and mechanical resonators, respectively; we have $\alpha=\beta=1$, consistent with the uncertainty relations and the canonical commutation relations of the noise operators. In what follows, we keep $\alpha$ and $\beta$ unspecified in order to clearly track the contributions of both mechanical and electromagnetic vacuum noise to the measured noise spectrum.

We include multiple bath temperatures $\left(n_{\sigma}^{\text {th }}\right)$ to describe the various sources of heating in microwave circuits. Compared to optical cavities which are passively cooled well into the ground state $\left(<10^{4} \mathrm{~K}\right)$, microwave cavities can have significant thermal occupation even at temperatures reached in the dilution refrigerator. Filtering on the input and output transmission lines suppresses incident roomtemperature noise; however, other issues may remain, like internal dissipation in the cavity [18] or thermal noise from refrigerator components. Additionally, there are other issues common to both microwave and optical systems, such as source-phase noise [19] and cavity-frequency jitter [20]. Whatever the source, noise in the system can be generalized into two categories based on how the noise contributes to the measured signal, either by radiating directly into the cavity $\left(n_{L}^{\text {th }}\right)$ or by radiating into both the cavity and detector $\left(n_{R}^{\text {th }}\right)$. For this experiment, $n_{R}^{\text {th }}$ describes noise generated from a hot circulator on the output line while $n_{L}^{\text {th }}$ describes all other significant noise sources.

We further specialize to the case where a single microwave-cavity drive is applied at $\omega_{p}=\omega_{c}-\Delta$ with $\Delta$ either $\pm \omega_{m}$, and consider the up- and down-converted sidebands generated by the mechanical motion. For simplicity, we ignore any internal loss of the cavity, consider the system to be in the sideband-resolved regime $\left(\kappa \ll \omega_{m}\right)$, and also consider the limit of a weak cooperativity, $\gamma_{\text {opt }}=4 G^{2} / \kappa \ll \gamma_{m}$. This last condition implies that the backaction effects on the mechanics are minimal: the mechanical linewidth and temperature are set by its coupling to its intrinsic dissipative bath.

There are several ways one could now measure the outgoing field $\hat{d}_{R, \text { out }}$ from the cavity and the corresponding 
power spectrum of its fluctuations. One general approach is to first measure the time-dependent quadrature amplitudes of the output field and then use these elements to calculate a power spectrum. We do this in our experiment by using a linear amplifier to measure the voltage associated with the outgoing field. In optics setups, it can be instead done using heterodyne detection, where one interferes the outgoing field with a reference beam. (This approach was used in Refs. $[14,15]$.) In both cases, one calculates the power spectrum from classical representations of quadrature amplitudes and is thus equally sensitive to absorption and emission of photons from this field. One thus necessarily measures a symmetrized amplitude power spectral density, which here takes the form

$$
\bar{S}_{I I, \text { tot }}[\omega]=\frac{1}{2} \int d t\left\langle\hat{I}_{\text {tot }}(t) \hat{I}_{\text {tot }}(0)+\hat{I}_{\text {tot }}(0) \hat{I}_{\text {tot }}(t)\right\rangle e^{i \omega t},
$$

with the amplitude of the output field $\hat{I}_{\text {tot }}=\hat{d}_{R \text {,out }}+\hat{d}_{R \text {,out }}^{\dagger}$ and where $\hat{d}_{R, \text { out }}=\hat{d}_{R, \text { in }}+\sqrt{\kappa_{R}} \hat{d}$. The output spectrum near the cavity resonance for the two choices of drive detuning are found to be

$$
\begin{aligned}
\left.\bar{S}_{I I, \text { tot }}[\omega]\right|_{\Delta=+\omega_{m}}= & \bar{S}_{0}+\frac{\kappa_{R}}{\kappa} \frac{\gamma_{\mathrm{opt}} \gamma_{m}}{\left(\frac{\gamma_{m}}{2}\right)^{2}+\left(\omega-\omega_{c}\right)^{2}} \\
& \times\left[\left(n_{m}^{\mathrm{th}}+\frac{\beta}{2}\right)-\left(n_{\mathrm{eff}}^{\mathrm{th}}+\frac{\alpha}{2}\right)\right], \\
\left.\bar{S}_{I I, \text { tot }}[\omega]\right|_{\Delta=-\omega_{m}}= & \bar{S}_{0}+\frac{\kappa_{R}}{\kappa} \frac{\gamma_{\mathrm{opt}} \gamma_{m}}{\left(\frac{\gamma_{m}}{2}\right)^{2}+\left(\omega-\omega_{c}\right)^{2}} \\
& \times\left[\left(n_{m}^{\mathrm{th}}+\frac{\beta}{2}\right)+\left(n_{\mathrm{eff}}^{\mathrm{th}}+\frac{\alpha}{2}\right)\right],
\end{aligned}
$$

where for $\Delta=\omega_{m}\left(\Delta=-\omega_{m}\right)$, the up- (down-) converted sideband is centered on the cavity resonance. The noise floor for both cases is given by $\bar{S}_{0}=\alpha / 2+$ $n_{R}^{\text {th }}+4 \kappa_{R}\left(n_{c}^{\text {th }}-n_{R}^{\text {th }}\right) / \kappa$, and we have defined $n_{\text {eff }}^{\text {th }}=2 n_{c}^{\text {th }}-$ $n_{R}^{\text {th }}$ [where $n_{c}^{\text {th }}=\left(\kappa_{L} n_{L}^{\text {th }}+\kappa_{R} n_{R}^{\text {th }}\right) / \kappa$ is the effective cavity thermal occupancy]. In Fig. 1(c), we illustrate the underlying components of this spectrum.

One sees explicitly that the sideband imbalance $\left.\bar{S}_{I I, \text { tot }}[\omega]\right|_{\Delta=-\omega_{m}}-\bar{S}_{I I, \text { tot }}[\omega] \|_{\Delta=+\omega_{m}}$ is proportional to $\left(2 n_{\mathrm{eff}}^{\text {th }}+\alpha\right)$ and hence is entirely due to fluctuations in the microwave fields driving the cavity. This interpretation is true both when this noise is thermal and when it is purely quantum (i.e., $n_{R}^{\text {th }}=n_{L}^{\text {th }}=0$ ). These terms in the spectrum result from the interference between the two ways the incident field noise can reach the output: either by directly being transmitted through the cavity or by first driving the mechanical resonator whose position then modulates the amplitude quadrature of the outgoing microwaves. (See Appendix A for further insights based on a scattering approach.) This interference is the basic mechanism of noise squashing, which in the case of thermal noise was

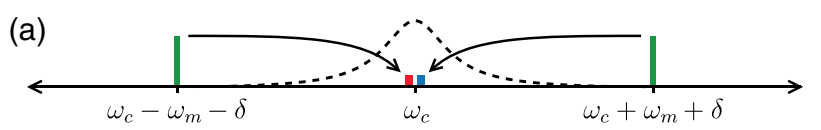

(b) Linear detection
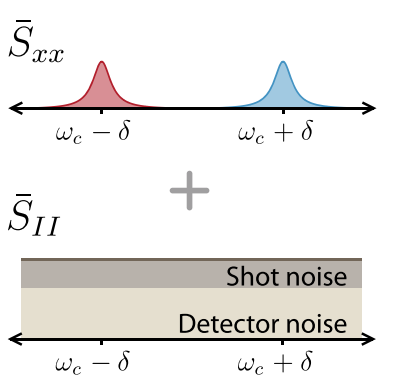

(c) Photodetection
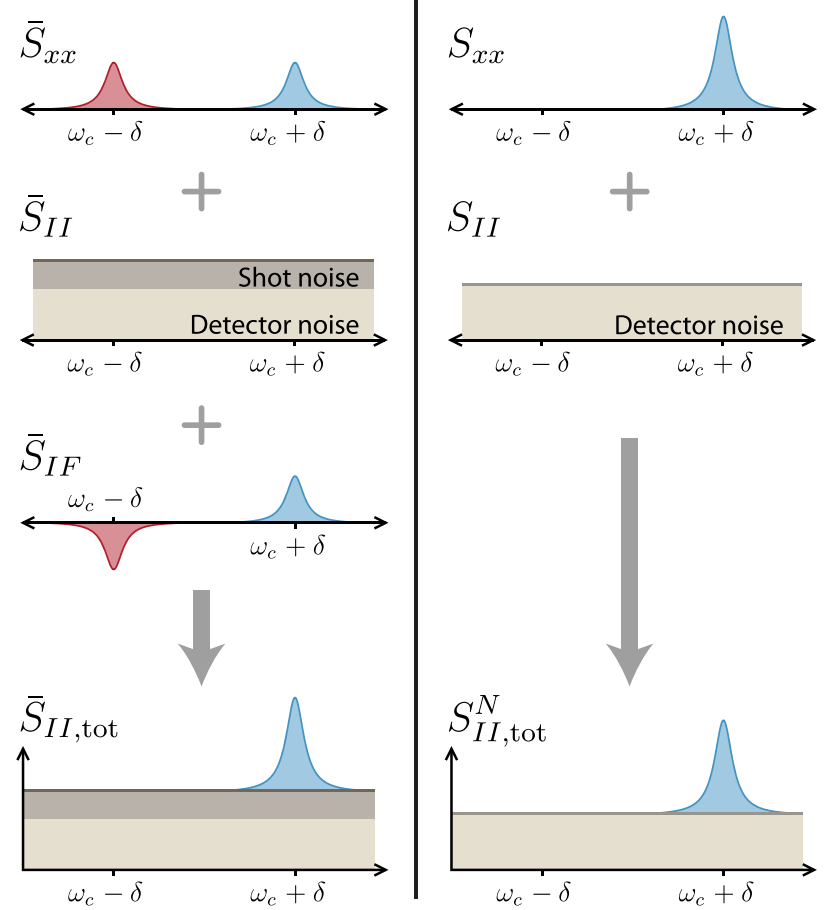

FIG. 1. Comparison between linear detection and photodetection. (a) Pump scheme. We consider a single microwave cavity (dotted line) pumped at $\omega_{c} \pm\left(\omega_{m}+\delta\right)$ (green bars). The upconverted (red bar) and down-converted (blue bar) motional sidebands are placed tightly within the cavity linewidth. For figure clarity, the occupations of the microwave and mechanical modes are assumed to be zero. (b) Linear detection. The quantum contribution from the symmetrized motional noise $\bar{S}_{x x}$ is present in both sidebands. Microwave shot noise (brown band) and amplifier noise (beige band) combine to form the imprecision noise $\bar{S}_{I I}$. This measurement is sensitive to noise correlation between the microwave and mechanical modes $\left(\bar{S}_{I F}\right)$, which results in asymmetric squashing (red region) and antisquashing (blue region) of the noise floor. (c) Photodetection. Normalordered detection is sensitive to the asymmetric motional noise spectrum $S_{x x}$. The detector is not sensitive to microwave shot noise, and the noise floor $\left(S_{I I}\right)$ is from detector nonidealities (beige band), analogous to dark counts for a photodetector. Although the source is different, the sideband imbalance is identical in both photodetection and linear detection. For mathematical descriptions of $S_{I I}$ and $S_{I F}$, refer to Appendix B.

previously observed in a microwave-cavity-based electromechanical system [4]. This mechanism can also be fully described using a general linear measurement formalism [16], where it is attributed to the presence of correlations between the backaction and imprecision noises of the detector, correlations that are out of phase and have magnitude $\hbar / 2$ in the zero-temperature limit. Interestingly, this precise value plays a special role in 
the theory of quantum limits on linear amplification [7]. (See Appendix B for more details.)

The above calculation also shows that both thermal and zero-point force noise emanating from the mechanical bath (i.e., terms $\propto n_{m}^{\text {th }}+\beta / 2$ ) contribute symmetrically to Eqs. (3) and (4) and hence play no role in determining the asymmetry of the sidebands. In the weak-cooperativity limit, it is the mechanical bath that almost entirely determines the mechanical oscillator fluctuations. This suggests that the sideband asymmetry observed using linear detection of the scattered field is not directly probing the asymmetric quantum noise spectrum of the mechanical mode.

An alternate measurement strategy to amplitude detection [which leads to Eq. (2)] is to first filter the output signal to a narrow bandwidth around a frequency $\omega$ and then perform direct photodetection. One is thus measuring power directly without first measuring field amplitudes, and in a manner that is only sensitive to the absorption of photons. As a result, such a measurement is described by the normal-ordered spectrum

$$
S_{I I, \text { tot }}^{\mathrm{N}}[\omega]=\int d t\left\langle\hat{d}_{R, \text { out }}^{\dagger}(0) \hat{d}_{R, \text { out }}(t)\right\rangle e^{i \omega t},
$$

with output spectra given by

$$
\begin{aligned}
\left.S_{I I, \text { tot }}^{\mathrm{N}}[\omega]\right|_{\Delta=+\omega_{m}}= & \left(\bar{S}_{0}-\frac{\alpha}{2}\right) \\
& +\frac{\kappa_{R}}{\kappa} \frac{\gamma_{\mathrm{opt}} \gamma_{m}}{\left(\frac{\gamma_{m}}{2}\right)^{2}+\left(\omega-\omega_{c}\right)^{2}}\left(n_{m}^{\mathrm{th}}-n_{\mathrm{eff}}^{\mathrm{th}}\right),
\end{aligned}
$$

$$
\begin{aligned}
\left.S_{I I, \mathrm{tot}}^{\mathrm{N}}[\omega]\right|_{\Delta=-\omega_{m}}= & \left(\bar{S}_{0}-\frac{\alpha}{2}\right) \\
& +\frac{\kappa_{R}}{\kappa} \frac{\gamma_{\mathrm{opt}} \gamma_{m}}{\left(\frac{\gamma_{m}}{2}\right)^{2}+\left(\omega-\omega_{c}\right)^{2}}\left(n_{m}^{\mathrm{th}}+\beta+n_{\mathrm{eff}}^{\mathrm{th}}\right) .
\end{aligned}
$$

Note that when one sets $\alpha=\beta=1$, the asymmetry of these normal-ordered spectra $\left.S_{I I, \text { tot }}^{\mathrm{N}}[\omega]\right|_{\Delta=-\omega_{m}}-\left.S_{I, \text { tot }}^{\mathrm{N}}[\omega]\right|_{\Delta=+\omega_{m}}$ is identical to that obtained from the linear measurement [where spectra are calculated using Eq. (2)]. In this case, however, the asymmetry is naturally attributed to both the mechanical quantum fluctuations $\beta$ and to the thermal microwave fluctuations described by $n_{\mathrm{eff}}^{\text {th }}$; this is illustrated in Fig. 1(b). Note that in ideal direct photodetection (i.e., no incident thermal fluctuations on the cavity), one cannot attribute the zero-temperature sideband asymmetry to a correlation between backaction-driven position fluctuations and imprecision noise, as there is no imprecision noise floor.

While the above simple calculations suggest that the sideband asymmetries measured using linear detection versus direct photodetection have different origins, it is no accident that the magnitudes of the asymmetry are the same in both schemes. This follows directly from the fact that the canonical commutation relation of the output field is the same as the input field $\left[\hat{d}_{R, \text { out }}(\omega), \hat{d}_{R, \text { out }}^{\dagger}\left(\omega^{\prime}\right)\right]=$ $\alpha \delta\left(\omega+\omega^{\prime}\right)$. It necessarily follows that the spectra in Eqs. (2) and (5) will differ only by a frequency-independent noise floor of magnitude $\alpha / 2[16,19]$. If one assumes this commutation relation, then one can legitimately say that both spectra essentially measure the same thing. However, on a formal level, this reasoning involves an additional assumption on the value of $\beta$ : if $\beta \neq \alpha$, then the output commutator would not be the same as the input commutator; see Appendix A.

Having explored the interpretation subtleties associated with sideband asymmetry, we now turn to presenting our main result: the experimental observation of this imbalance in a microwave-cavity-based electromechanical system.

\section{RESULTS}

Our system is composed of a superconducting microwave resonator, also referred to as a "cavity," where the resonance frequency is modulated by the motion of a compliant membrane [13]. This frequency modulation leads to the desired parametric coupling between microwave field and mechanical motion [Fig. 2(a)]. Measurements of the cavity response below $100 \mathrm{mK}$ yield the resonance frequency $\omega_{c}=2 \pi \times 5.4 \mathrm{GHz}$, total loss rate $\kappa=2 \pi \times 860 \mathrm{kHz}$, output coupling rate $\kappa_{R}=2 \pi \times$ $450 \mathrm{kHz}$, and input coupling rate $\kappa_{L}=2 \pi \times 150 \mathrm{kHz}$. The capacitor top gate is a flexible aluminum membrane $(40 \mu \mathrm{m} \times 40 \mu \mathrm{m} \times 150 \mathrm{~nm})$ with a fundamental drumhead mode with resonance frequency $\omega_{m}=2 \pi \times 4.0 \mathrm{MHz}$ and intrinsic loss rate $\gamma_{m}=2 \pi \times 10 \mathrm{~Hz}$ at $20 \mathrm{mK}$. Motional displacement of the top gate modulates the microwaveresonance frequency with an estimated coupling rate of $g_{0}=\left(\partial \omega_{c} / \partial x\right) x_{\mathrm{ZP}}=2 \pi \times 16 \mathrm{~Hz}$.

In Fig. 2(c), we present a schematic of the measurement circuit. Tunable cavity filters at room temperature reduce the source-phase noise to the thermal noise level at $300 \mathrm{~K}$; cryogenic attenuators further reduce the noise down to the shot noise level [4]. A pair of microwave switches at the device stage selects between the device or a bypass connection for high precision noise floor calibration of the cryogenic amplifier. The output signal passes through two cryocirculators at approximately $100 \mathrm{mK}$, followed by a cryogenic high-electron-mobility transistor amplifier (HEMT) at $4.2 \mathrm{~K}$, and finally to room-temperature circuits for analysis. The occupation factor of the microwave resonator $n_{c}^{\text {th }}$, which is expected to thermalize below $5 \times 10^{-3}$ at temperatures below $50 \mathrm{mK}$, can be increased and controlled by the injection of microwave-frequency noise from amplified room-temperature Johnson noise. From careful measurements of the noise power emanating from the cavity at zero pumping compared to power spectra with the bypass switched in place, we conclude that there is a small contribution to $n_{c}^{\text {th }}$ due to thermal radiation from the 


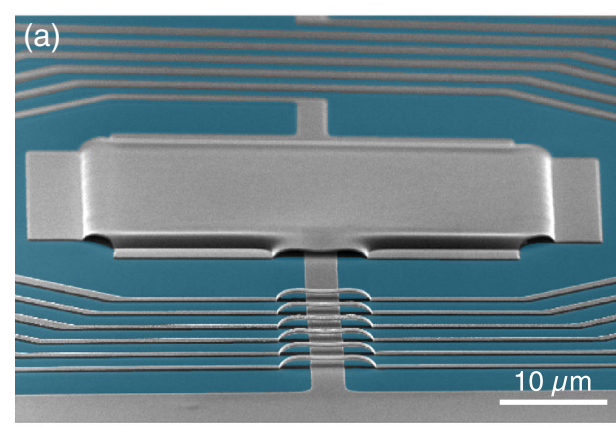

(c)
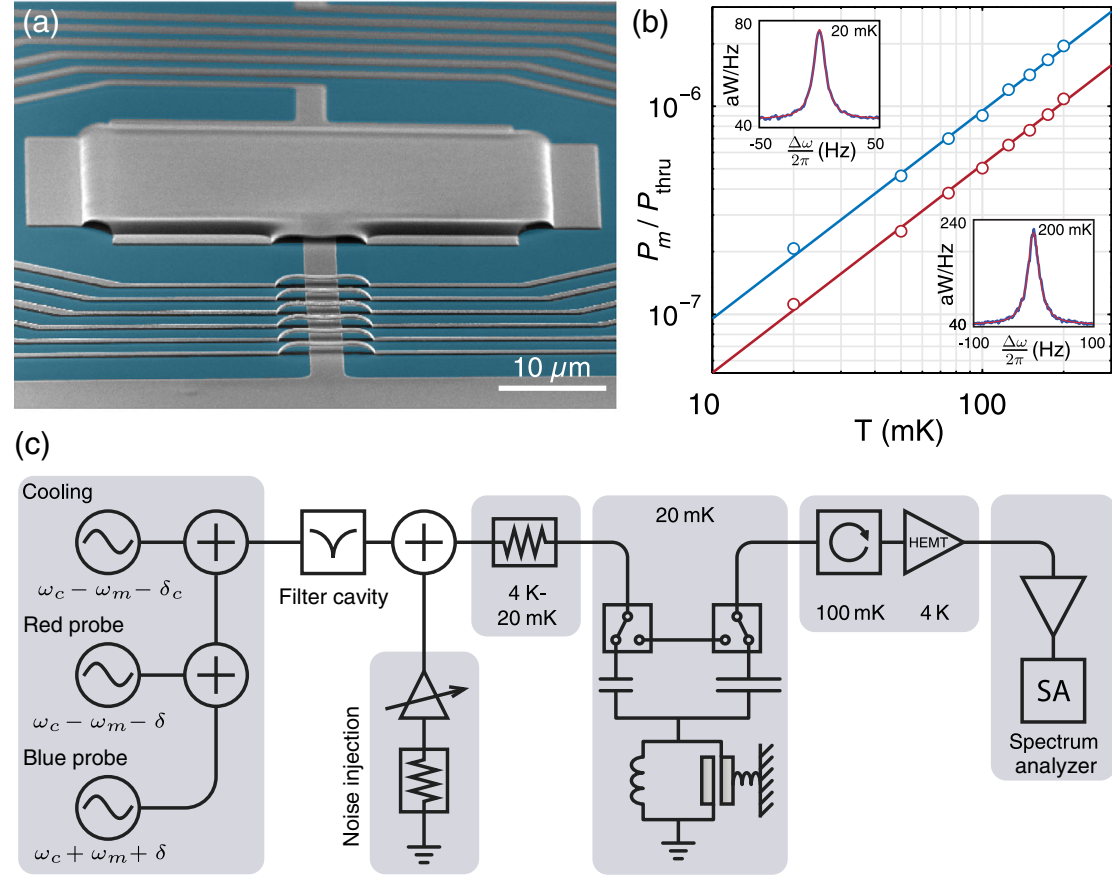

FIG. 2. Device, calibration, and measurement scheme. (a) Electron micrograph of the measured device. A suspended aluminum (gray areas) membrane patterned on silicon (blue background) forms the electromechanical capacitor. It is connected to the surrounding spiral inductor to form a microwave resonator. Out of view, coupling capacitors on either side of the inductor couple the device to input and output coplanar waveguides. (b) Motional sideband calibration. The cryostat temperature is regulated while the mechanical mode is weakly probed with microwave tones set at $\omega_{c}+\omega_{m}+\delta$ (blue) and at $\omega_{c}-\omega_{m}-\delta$ (red), with detunings $\delta=2 \pi \times 500 \mathrm{~Hz}$. The observed linear dependence provides the calibration between the normalized sideband power and the mechanical occupation factor. Insets: Up-converted motional sideband spectra collected at $20 \mathrm{mK}$ (top) and $200 \mathrm{mK}$ (bottom), with $\Delta \omega=\omega-\left(\omega_{c}-\delta\right.$ ). (c) Schematic of the microwave-measurement circuit.

isolated port of the cryogenic circulators, given by the occupation factor $n_{R}^{\text {th }}=0.25 \pm 0.03$ [21]. Since performing this experiment, we have reduced the temperature of the circulator and have decreased the output-port occupation below measurement sensitivity levels $\left(n_{R}^{\text {th }} \leq 0.03\right)$.

When a single microwave tone is applied to the device at $\omega_{p}$, the parametric coupling converts mechanical oscillations at $\omega_{m}$ to up- and down-converted sidebands at $\omega_{p} \pm \omega_{m}$. In this experiment, we apply microwave tones at frequencies near $\omega_{c} \pm \omega_{m}$ and at powers given by the mean number of photons in the resonator $n_{p}$. The microwave resonance suppresses motional sidebands outside of the linewidth, and we consider only the contributions of signals converted to frequencies near $\omega_{c}$. These sidebands are the Lorentzian components of the noise spectra of Eqs. (3) and (4), which for the remainder of the paper are denoted by plus and minus signs, respectively.

Throughout the measurement, we simultaneously apply three microwave tones. We place a cooling tone at $\omega_{c}-$ $\omega_{m}-\delta_{c}$ to control the effective mechanical damping rate $\gamma_{M}$ and mode occupation $\bar{n}_{m}$ via backaction cooling [22,23]. Two additional probe tones, placed at $\omega_{c} \pm\left(\omega_{m}+\delta\right)$, produce up- and down-converted sidebands symmetrically detuned from the cavity center [Fig. 3(a)]. The detunings are chosen to ensure no interference between the sidebands $\left(\delta_{c}=2 \pi \times 30 \mathrm{kHz}, \delta=2 \pi \times 5 \mathrm{kHz}\right)$ so that we may consider the probe sidebands as independent measurements of the dressed mechanical mode.

There are several differences between the theory model developed above and the experimental realization described here. In practice, we measure the mechanical sidebands produced in a two-port microwave resonator with limited sideband resolution and a noisy output port, and in the presence of multiple injected tones with a range of detunings and powers. From further analysis of the experimental configuration (see Appendix A 2), we estimate corrections to Eqs. (3) and (4) that are $\ll 1$ and far below the measurement resolution of our system.

To convert the motional sideband powers into equivalent mechanical occupation $\left(\bar{n}_{m}\right)$, we turn off the cooling tone and measure the probe sidebands $(\delta=2 \pi \times 500 \mathrm{~Hz})$ with low optical damping $\left(n_{p}^{+}=n_{p}^{-} \simeq 5 \times 10^{2}\right)$ and high mechanical occupation set by the cryostat temperature. Such low pump powers ensure that the mechanical sideband signals are dominated solely by the intrinsic thermal noise of the mechanical mode; other effects such as dynamical backaction, cavity-noise interference, or mechanical bath heating are negligible in this regime $[20,21]$. Regulating the temperature to calibrated levels between 20 and $200 \mathrm{mK}$, we calculate the integrated noise 

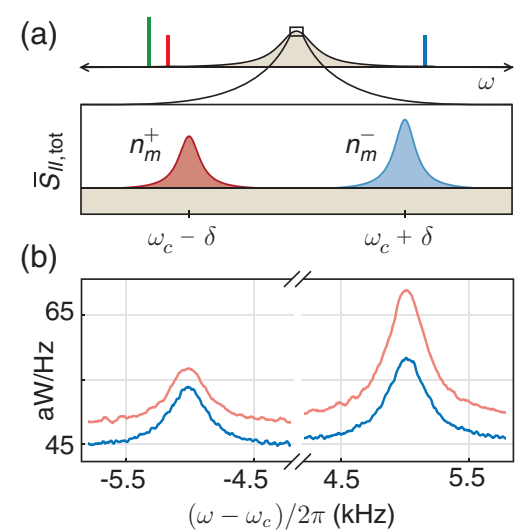

(c)

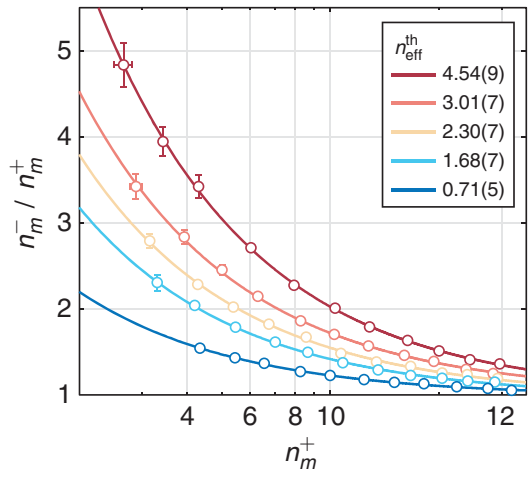

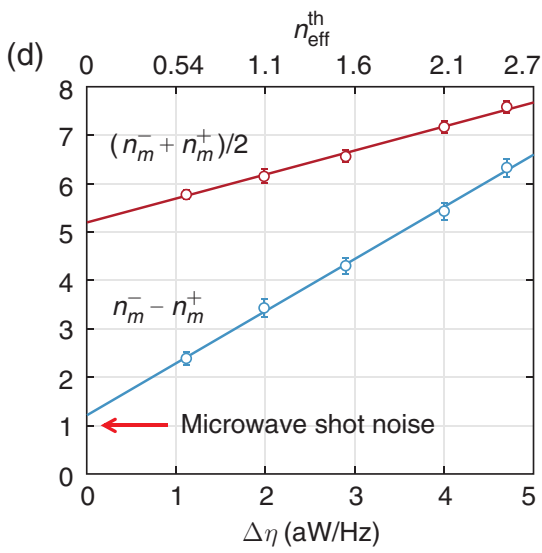

FIG. 3. Sideband asymmetry. (a) Pump scheme. Three tones are placed about the microwave resonance. Two probe tones generate upconverted (red) and down-converted (blue) sidebands. An additional tone (green) cools the mechanical mode. (b) Sideband spectra. $\bar{S}_{I I, \text { tot }}[\omega]$ measured at $n_{\mathrm{eff}}^{\text {th }}=0.60$ (blue) and 2.5 (orange) with $\bar{n}_{m}=5.3$ and 7.1, respectively. (c) Sideband asymmetry. The ratio $n_{m}^{-} / n_{m}^{+}$ versus $n_{m}^{+}$is plotted for increasing noise injection. (d) Sideband imbalance (blue) and sideband average (red) versus the measured noise increase $\Delta \eta$. Sideband imbalance $n_{m}^{-}-n_{m}^{+}$and average $\left(n_{m}^{-}+n_{m}^{+}\right) / 2$ exhibit a linear trend with $\Delta \eta$. The imbalance at $\Delta \eta=0$ is the quantum imbalance due to the squashing of fluctuations of the microwave field.

power under the sideband Lorentzians $P_{m}^{ \pm}$, normalized by the respective microwave-probe power transmitted through the device $P_{\mathrm{thru}}^{ \pm}$. In the limit of high thermal occupation, the normalized power is directly proportional to $\bar{n}_{m}$ [24]. As we vary the cryostat temperature $T$, we compare the normalized power to the thermal occupation factor $\left[\exp \left(\hbar \omega_{m} / k_{B} T\right)-1\right]^{-1}$ [Fig. 2(b)]. A linear fit yields the conversion factors for the up-converted $\left(n_{m}^{+}\right)$and down-converted $\left(n_{m}^{-}\right)$sidebands: $n_{m}^{+}=(9.9 \pm 0.2) \times 10^{8} P_{m}^{+} / P_{\text {thru }}^{+}$and $n_{m}^{-}=(5.4 \pm 0.1) \times$ $10^{8} P_{m}^{-} / P_{\text {thru }}^{-}$. The factor of approximately 2 between calibration factors at the two pump detunings is due to the presence of a parasitic bypass channel in the microwave circuit that allows pump signals to weakly transmit across the input and output ports of the device while completely bypassing the microwave resonator. (See Sec. 4 of Ref. [21].)

Further detuning the probe tones $(\delta=2 \pi \times 5 \mathrm{kHz})$ and turning on the cooling tone $\left(\delta_{c}=2 \pi \times 30 \mathrm{kHz}\right)$, we explore the sideband ratio $n_{m}^{-} / n_{m}^{+}$over various mechanical and microwave occupations. To reduce $\bar{n}_{m}$ to values approaching 1, we increase the cooling-tone power up to $n_{p}^{\text {cool }}=4 \times 10^{5}$. For sideband characterization, the probetone powers are set to $n_{p}^{-}=n_{p}^{+}=10^{5}$ in order to achieve sufficient measurement sensitivity and the probe sideband spectra are analyzed using the conversion factors described above. The imbalance between $n_{m}^{-}$and $n_{m}^{+}$is clearly evident in the noise spectra [Fig. 3(b)].

As further demonstration of the asymmetry with respect to $n_{\mathrm{eff}}^{\text {th }}$, we plot the ratio $n_{m}^{-} / n_{m}^{+}$as a function of $n_{m}^{+}$in Fig. 3(c). Each curve corresponds to one setting of injected microwave noise. The data show excellent agreement to the expected ratio $n_{m}^{-} / n_{m}^{+}=1+\left(2 n_{\mathrm{eff}}^{\text {th }}+1\right) / n_{m}^{+}$. This relationship highlights the combined effect of quantum and classical noise in Eqs. (3) and (4). By fitting each curve to a two-parameter model $a+b / n_{m}^{+}$, we find an average constant offset $a=0.99 \pm 0.02$ for all curves, accurately matching the model and confirming our calibration techniques. Fitting for $b$, the data indicate $n_{\mathrm{eff}}^{\text {th }}$, spanning 0.71 to 4.5 , with uncertainty all within \pm 0.09 quanta.

To quantify the contributions due to quantum fluctuations and classical cavity noise, we fix the cooling-tone power at $n_{p}^{\text {cool }}=4 \times 10^{5}\left(\gamma_{M}=2 \pi \times 360 \mathrm{~Hz}\right)$ and measure the imbalance $n_{m}^{-}-n_{m}^{+}$as we sweep $n_{\mathrm{eff}}^{\text {th }}$. At each level, we measure the average noise-power density $\eta$ over a $250-\mathrm{Hz}$ window centered at $\omega_{c}$ and away from any motional sideband. Over this range, $\eta$ contains two contributions: the noise radiating out of the microwave resonator, proportional to $n_{\mathrm{eff}}^{\text {th }}$, and the detector noise floor, set by the noise temperature of the cryogenic amplifier $\left(T_{N} \approx 3.6 \mathrm{~K}\right)$. We directly measure the detector noise floor by switching from the device to an impedance-matched bypass connection and measure the noise-power density $\eta_{0}$ over the same window with matching detected tone powers.

In Fig. 3(d), we plot the sideband imbalance against the noise floor increase $\Delta \eta=\eta-\eta_{0}$, which is expected to follow $n_{m}^{-}-n_{m}^{+}=2 n_{\text {eff }}^{\text {th }}+1=4 \lambda \Delta \eta+1$, where $\lambda$ is the conversion factor for $\Delta \eta$ in units of cavity quanta $n_{c}^{\text {th }}$. The data clearly follow a linear trend with a slope of $\lambda=(2.7 \pm 0.1) \times 10^{-1}(\mathrm{aW} / \mathrm{Hz})^{-1}$. More importantly, we observe an offset of $1.2 \pm 0.2$, in excellent agreement with the expected quantum imbalance of +1 from the quantum fluctuations of the microwave field.

As an additional check, we also consider the sideband average $\left(n_{m}^{+}+n_{m}^{-}\right) / 2$ as a function of $\Delta \eta$. Averaging Eqs. (3) and (4), we see that the resulting occupation $\bar{n}_{m}+$ $(\beta / 2)$ does depend on $n_{\mathrm{eff}}^{\text {th }}$ due to the coupling between the mechanical and microwave modes, $\bar{n}_{m}=\left(\gamma_{m} / \gamma_{\text {tot }}\right) n_{m}^{\text {th }}+$ $\left(\gamma_{\mathrm{opt}} / \gamma_{\mathrm{tot}}\right)\left(2 n_{c}^{\text {th }}+\alpha\right)+\left(\gamma_{\mathrm{opt}}^{\mathrm{cool}} / \gamma_{\mathrm{tot}}\right) n_{c}^{\text {th }}$, where $\gamma_{\mathrm{opt}}\left(\gamma_{\mathrm{opt}}^{\mathrm{cool}}\right)$ is the optical coupling rate for the individual probe (cooling) 
tones. Accounting for this so-called backaction heating of the mechanical mode [13,22], we recover $\lambda=(2.5 \pm 0.2) \times$ $10^{-1}(\mathrm{aW} / \mathrm{Hz})^{-1}$, consistent with the imbalance results above.

Notably, the average sideband occupation does contain contributions from mechanical zero-point fluctuations. Future experiments could infer the mechanical quantum contribution of $(\beta / 2)$ with a method to independently calibrate $\bar{n}_{m}$ to high accuracy, for example, with a passively cooled high-frequency mechanical mode thermalized to a primary low-temperature thermometer.

In summary, we report the quantum imbalance between the up- and down-converted motional sideband powers in a cavity-electromechanical system measured with a symmetric, linear detector. We show that for linear detection of the microwave field, the imbalance arises from the correlations between the mechanical motion and the quantum fluctuations of the microwave field. For normal-ordered detection of the microwave field, however, the imbalance arises directly from the quantum fluctuations of the mechanics. By further assuming that the output microwave field satisfies the canonical commutator, which also determines the quantum fluctuations of the mechanical mode, the measurement can be interpreted as performing either symmetric or normal-ordered detection regardless of the type of detector utilized. In either interpretation, the imbalance in motional sideband power stems from two components: the quantum fluctuations of the internal fields and the classical thermal noise from the environment. Once the classical contribution is reduced or calibrated to well below the quantum noise level, sideband imbalance provides a quantum-calibrated thermometer for mesoscopic mechanical systems.

\section{ACKNOWLEDGMENTS}

We would like to acknowledge Yanbei Chen and Matthew Woolley for helpful discussions. This work is supported by funding provided by the Institute for Quantum Information and Matter, an NSF Physics Frontiers Center with support of the Gordon and Betty Moore Foundation (NSF-IQIM 1125565), by DARPA (DARPA-QUANTUM HR0011-10-1-0066), by the NSF (NSF-DMR 1052647 and NSF-EEC 0832819), and by the DARPA ORCHID Program under a grant from AFOSR.

Note added.-Recently, two other groups have reported similar asymmetry measurements [25,26]. Both groups have used linear measurement schemes and are subject to the same interpretation issues described here.

\section{APPENDIX A: INPUT-OUTPUT THEORY}

In this section, we give a framework to calculate the output-noise spectrum of an opto- or electromechanical system with arbitrary pump configuration by utilizing the input-output theory. As a first example, we analyze an ideal (without intrinsic losses) two-port opto- or electromechanical system with a single pump tone either at frequency $\omega_{p}=\omega_{c}-\omega_{m}$ or $\omega_{p}=\omega_{c}+\omega_{m}$ and discuss the origin of the sideband asymmetry in the output-noise spectrum. We then use this method to study the system in our experiment, i.e., a two-port electromechanical system with three pumps (two balanced detuned tones and a cooling tone).

We start with the standard Hamiltonian of an opto- or electromechanical system

$$
\begin{aligned}
\hat{\mathcal{H}}= & \hbar \omega_{c} \hat{a}^{\dagger} \hat{a}+\hbar \omega_{m} \hat{b}^{\dagger} \hat{b}+\hbar g_{0} \hat{a}^{\dagger} \hat{a}\left(\hat{b}+\hat{b}^{\dagger}\right) \\
& +\hat{\mathcal{H}}_{\text {drive }}+\hat{\mathcal{H}}_{\text {diss }},
\end{aligned}
$$

where $\hat{a}\left(\hat{a}^{\dagger}\right)$ is the annihilation (creation) operator of the cavity field. $\hat{b}\left(\hat{b}^{\dagger}\right)$ is the annihilation (creation) operator of the phonon, and $g_{0}$ is the coupling strength between the cavity and the mechanical oscillator. We assume an external driving, described by $\hat{\mathcal{H}}_{\text {drive }}$, which is applied on the input port on the left side of the cavity. The optical and the mechanical systems are both coupled to dissipative baths, described by $\hat{\mathcal{H}}_{\text {diss }}$, giving rise to the decay rates $\gamma_{m}$ for the mechanical and $\kappa$ for the optical systems. The total cavity linewidth $\kappa$ consists of the contributions from the different decay channels, namely, the right $(R)$ and the left $(L)$ ports, as well as from intrinsic losses $(I)$ inside the cavity, i.e., $\kappa=\kappa_{R}+\kappa_{L}+\kappa_{I}$.

For large pumping fields, we may split the fields into classical and quantum components $\hat{a} \rightarrow \bar{a}+\hat{d}$ and $\hat{b} \rightarrow \bar{b}+\hat{c}$, where $\hat{d}$ and $\hat{c}$ describe the quantum fluctuations of the cavity photon and the phonon. By using input-output theory and neglecting the second-order contributions from the quantum fluctuations, the linearized quantum Langevin equations are

$$
\begin{aligned}
& \dot{\hat{d}}=-i \omega_{c}^{\prime} \hat{d}-\frac{\kappa}{2} \hat{d}-i g_{0} \bar{a}(t)\left[\hat{c}+\hat{c}^{\dagger}\right]-\sum_{\sigma \in L, R, I} \sqrt{\kappa_{\sigma}} \hat{d}_{\sigma, \mathrm{in}}, \\
& \dot{\hat{c}}=-i \omega_{m} \hat{c}-\frac{\gamma_{m}}{2} \hat{c}-i g_{0}\left[\bar{a}^{*}(t) \hat{d}+\bar{a}(t) \hat{d}^{\dagger}\right]-\sqrt{\gamma_{m}} \hat{c}_{\mathrm{in}},
\end{aligned}
$$

where $\omega_{c}^{\prime}=\omega_{c}+g\left(\bar{b}+\bar{b}^{*}\right) \simeq \omega_{c}$. Including the possibility of multiple drives at frequencies $\omega_{n}$, we obtain $\bar{a}(t)=$ $\sum_{n} \bar{a}_{n} e^{-i \omega_{n} t}$ as the driving field inside the cavity, with $\bar{a}_{n}=\sqrt{\kappa_{L}} \alpha_{n} /\left[(\kappa / 2)-i\left(\omega_{n}-\omega_{c}\right)\right]$. Without loss of generality, we take $\bar{a}_{n}$ to be real. In Eqs. (A2), $\hat{d}_{\sigma \text {,in }}$ describes the input fluctuations to the cavity from channel $\sigma$ with damping rate $\kappa_{\sigma}$, and $\hat{c}_{\text {in }}$ describes the input fluctuations to the mechanical oscillator. The input field operators satisfy the following commutation relations: 


$$
\begin{aligned}
{\left[\hat{d}_{\sigma, \text { in }}(t), \hat{d}_{\sigma^{\prime} \text { in }}^{\dagger}\left(t^{\prime}\right)\right] } & =\alpha_{\sigma} \delta_{\sigma \sigma^{\prime}} \delta\left(t-t^{\prime}\right), \\
{\left[\hat{c}_{\text {in }}(t), \hat{c}_{\text {in }}^{\dagger}\left(t^{\prime}\right)\right] } & =\beta \delta\left(t-t^{\prime}\right), \\
\left\langle\hat{d}_{\sigma, \text { in }}^{\dagger}(t) \hat{d}_{\sigma^{\prime} \text { in }}\left(t^{\prime}\right)\right\rangle & =n_{\sigma}^{\text {th }} \delta_{\sigma \sigma^{\prime}} \delta\left(t-t^{\prime}\right), \\
\left\langle\hat{c}_{\text {in }}^{\dagger}(t) \hat{c}_{\text {in }}\left(t^{\prime}\right)\right\rangle & =n_{m}^{\text {th }} \delta\left(t-t^{\prime}\right),
\end{aligned}
$$

where $\alpha_{\sigma}=\beta=1, n_{\sigma}^{\text {th }}$ is the photon occupation in port $\sigma$, and $n_{m}^{\text {th }}=1 /\left[\exp \left(\hbar \omega_{m} / k_{B} T\right)-1\right]$ is the thermal occupation factor of the bath responsible for the intrinsic mechanical dissipation. The total thermal occupation of the cavity is the weighted sum of the contributions from different channels $n_{c}^{\text {th }}=\sum_{\sigma}\left(\kappa_{\sigma} / \kappa\right) n_{\sigma}^{\text {th }}$. Note that the relations in Eq. (A3) are only valid if we deal with frequencies close to cavity resonance.

\section{Single tone}

We start with the case of a single pump tone at frequency $\omega_{p}=\omega_{c}-\Delta$, where the drive detuning $\Delta$ is chosen to

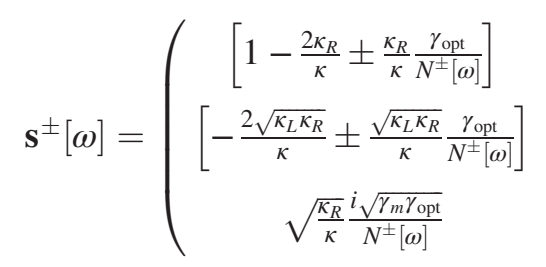

Here, the denominator $N^{ \pm}[\omega]$ describes the mechanical response including optical damping or antidamping:

$$
N^{ \pm}[\omega]=-i\left(\omega \mp \omega_{m}\right)+\frac{\gamma_{m} \pm \gamma_{\mathrm{opt}}}{2}, \quad \gamma_{\mathrm{opt}}=\frac{4 G^{2}}{\kappa},
$$

with $G=g_{0} \bar{a}_{p}$ being the many-photon optomechanical coupling rate.

Our interest is in the output field leaving the right port of the cavity and hence in the first row of $\mathbf{s}^{ \pm}[\omega]$. For a weak optomechanical cooperativity, we can ignore the modification of the mechanical damping by the cavity and approximate $\gamma_{m} \pm \gamma_{\mathrm{opt}} \simeq \gamma_{m}$. The only remaining differences in the first row of $\mathbf{s}^{+}$versus $\mathbf{s}^{-}$are in the overall sign of the mechanical contributions (terms $\propto \gamma_{\text {opt }}$ ) in the elements $\mathbf{s}_{11}$ and $\mathbf{s}_{12}$. These elements describe how the incident microwave fluctuations show up in the output; the sign difference of the mechanical term directly mirrors the fact that for the red- (blue-) detuned drive, the cavity provides positive (negative) optical damping on the mechanics. Note, finally, that for weak coupling, the coefficient $\mathbf{s}_{13}$ describing the transmission of mechanical bath fluctuations to the output is identical for both choices of drive detuning.

The normal-ordered noise spectral density and the symmetrized noise spectral density of the output field on the right side of the cavity are defined as either be $\pm \omega_{m}$; our goal is to make the origin of the asymmetry between the spectra measured for these two cases clear. For maximum clarity, we also consider the good-cavity limit $\omega_{m} \gg \kappa$ and work within the rotatingwave approximation (RWA). In this limit, we can describe the relevant spectra in terms of a $3 \times 3$ scattering matrix, involving the fields $\hat{\mathbf{D}}^{+(-)} \equiv\left(\hat{d}_{R}, \hat{d}_{L}, \hat{c}^{(\dagger)}\right)^{T}$, where the plus sign (minus sign) refers to a driving on the red (blue) sideband, i.e., $\Delta= \pm \omega_{m}$. By using the input-output relations $\hat{d}_{\sigma, \text { out }}=\hat{d}_{\sigma \text {,in }}+\sqrt{\kappa_{\sigma}} \hat{d}_{\sigma}$ and $\hat{c}_{\text {out }}=\hat{c}_{\text {in }}+\sqrt{\gamma_{m}} \hat{c}$ and solving the corresponding quantum Langevin equations, we obtain in frequency space (working in a rotating frame at the cavity frequency) [27]

$$
\hat{\mathbf{D}}_{\text {out }}^{ \pm}[\omega]=\mathbf{s}^{ \pm}[\omega] \hat{\mathbf{D}}_{\text {in }}^{ \pm}[\omega] .
$$

For frequencies close to the cavity resonance (i.e., $|\omega-\Delta| \ll \kappa)$, the scattering matrix $\mathbf{s}[\omega]$ is

$$
\begin{aligned}
& S_{I I, \text { tot }}^{\mathrm{N}}[\omega]=\int d t\left\langle\hat{d}_{R, \text { out }}^{\dagger}(0) \hat{d}_{R, \text { out }}(t)\right\rangle e^{i \omega t}, \\
& \bar{S}_{I I, \text { tot }}[\omega]=\frac{1}{2} \int d t \overline{\langle\{\hat{I}(t+\bar{t}), \hat{I}(\bar{t})\}\rangle} e^{i \omega t},
\end{aligned}
$$

where $\hat{I}(t)=\hat{d}_{R \text {,out }}(t)+\hat{d}_{R \text {,out }}^{\dagger}(t)$ and all operators are lab frame output operators. In the definition of the symmetrized correlator, the bar indicates an average over the center-ofmass time $\bar{t}$, which implies that we only characterize the stationary aspects of the output noise. The above definition is consistent with the symmetrized noise spectral density measured by a classical voltage-spectrum analyzer, as used in the experiment. In the simple RWA theory presented in the main text, the symmetrized correlation function is explicitly independent of $\bar{t}$. This is no longer true when one considers non-RWA corrections, or multi-tone driving (although the $\bar{t}$-dependent terms remain extremely small for relevant parameters).

A further simplification arises from the fact that we focus on frequencies near the cavity-resonance frequency (i.e., $\omega \simeq \omega_{c}$ in the lab frame). For such frequencies, terms in the spectra involving the output operator $\hat{d}_{R, \text { out }}^{\dagger}(t+\bar{t})$ will not contribute, as these operators only have spectral weight at negative frequencies in the lab frame (see, e.g., Appendix D in Ref. [7].) We can thus replace $\{\hat{I}(t+\bar{t})$, $\hat{I}(\bar{t})\}$ by $\left\{\hat{d}_{R, \text { out }}(t+\bar{t}), \hat{d}_{R, \text { out }}^{\dagger}(\bar{t})\right\}$ in the definition of the symmetrized spectrum. 
Having established the definition of the noise spectra, we now return to our rotating frame, where the cavity frequency is situated at $\omega=\Delta$. By using the correlators defined in Eq. (A3), we can calculate these noise spectral densities and express them in terms of the elements of the scattering matrix [Eq. (A5)]. We obtain for the symmetrized spectra

$$
\begin{aligned}
\left.\bar{S}_{I I, \text { tot }}[\omega]\right|_{\Delta= \pm \omega_{m}}= & \left|s_{11}^{ \pm}[\omega]\right|^{2}\left(n_{R}^{\text {th }}+\frac{\alpha_{R}}{2}\right) \\
& +\left|s_{12}^{ \pm}[\omega]\right|^{2}\left(n_{L}^{\text {th }}+\frac{\alpha_{L}}{2}\right) \\
& +\left|s_{13}^{ \pm}[\omega]\right|^{2}\left(n_{m}^{\text {th }}+\frac{\beta}{2}\right),
\end{aligned}
$$

while the normal-ordered spectra take the form

$$
\begin{aligned}
\left.S_{I I, \text { tot }}^{\mathrm{N}}[\omega]\right|_{\Delta=+\omega_{m}}= & \left|s_{11}^{+}[\omega]\right|^{2} n_{R}^{\text {th }}+\left|s_{12}^{+}[\omega]\right|^{2} n_{L}^{\text {th }} \\
& +\left|s_{13}^{+}[\omega]\right|^{2} n_{m}^{\text {th }}, \\
\left.S_{I I, \text { tot }}^{\mathrm{N}}[\omega]\right|_{\Delta=-\omega_{m}}= & \left|s_{11}^{-}[\omega]\right|^{2} n_{R}^{\text {th }}+\left|s_{12}^{-}[\omega]\right|^{2} n_{L}^{\text {th }} \\
& +\left|s_{13}^{-}[\omega]\right|^{2}\left(n_{m}^{\text {th }}+\beta\right) .
\end{aligned}
$$

Note crucially that for a given drive detuning, the scattering-matrix elements appear identically in both the symmetrized and normal-ordered spectra. The only difference is how these elements are weighted by the input noise. For the symmetrized spectra, it is always the symmetrized bath noise that enters (i.e., $n_{\sigma}^{\text {th }}+1 / 2$ ), irrespective of the drive detuning. In the normal-ordered case, we see that the only contribution from vacuum noise is from the mechanical bath, and only for the case of a blue-detuned drive. We also note that the form of the symmetrized spectra given above could be obtained from a completely classical set of Langevin equations, as the input-noise correlators enter the same way for both detunings. This is not true for the normal-ordered case, as the effective mechanical bath correlator is different for $\Delta=\omega_{m}$ versus $\Delta=-\omega_{m}$.

Setting $\alpha_{R}=\alpha_{L} \equiv \alpha$ for clarity, the imbalance of the spectra (i.e., the difference between the output spectra for the two choices of detuning) $\delta S=\left.S\right|_{\Delta=-\omega_{m}}-\left.S\right|_{\Delta=\omega_{m}}$ become

$$
\begin{aligned}
& \delta \bar{S}_{I I, \text { tot }}=\left\{\left|s_{11}^{-}\right|^{2}-\left|s_{11}^{+}\right|^{2}\right\}\left(n_{R}^{\text {th }}+\frac{\alpha}{2}\right)+\left\{\left|s_{12}^{-}\right|^{2}-\left|s_{12}^{+}\right|^{2}\right\}\left(n_{L}^{\text {th }}+\frac{\alpha}{2}\right)+\left\{\left|s_{13}^{-}\right|^{2}-\left|s_{13}^{+}\right|^{2}\right\}\left(n_{m}^{\text {th }}+\frac{\beta}{2}\right), \\
& \delta S_{I I, \text { tot }}^{\mathrm{N}}=\left\{\left|s_{11}^{-}\right|^{2}-\left|s_{11}^{+}\right|^{2}\right\} n_{R}^{\text {th }}+\left\{\left|s_{12}^{-}\right|^{2}-\left|s_{12}^{+}\right|^{2}\right\} n_{L}^{\text {th }}+\left\{\left|s_{13}^{-}\right|^{2}-\left|s_{13}^{+}\right|^{2}\right\} n_{m}^{\text {th }}+\left|s_{13}^{-}\right|^{2} \beta .
\end{aligned}
$$

We have omitted writing the explicit frequency dependence of the elements of $\mathbf{s}^{ \pm}$for clarity.

Finally, we insert the explicit elements of the scattering matrix in Eq. (A5) into the expressions for the different output spectra derived above. The symmetrized noise in the rotating frame becomes

$$
\begin{aligned}
& \left.\bar{S}_{I I, \text { tot }}[\omega]\right|_{\Delta=+\omega_{m}}=\bar{S}_{0}+\frac{\kappa_{R}}{\kappa} \frac{\gamma_{m} \gamma_{\mathrm{opt}}}{\left(\omega-\omega_{m}\right)^{2}+\frac{\gamma_{\mathrm{tot}}^{2}}{4}}\left\{n_{m}^{\mathrm{th}}-n_{\mathrm{eff}}^{\mathrm{th}}+\frac{\beta-\alpha_{R}}{2}-\frac{\gamma_{\mathrm{opt}}}{\gamma_{m}}\left[n_{c}^{\mathrm{th}}-n_{R}^{\mathrm{th}}\right]-\frac{\kappa_{\mathrm{L}}}{\kappa}\left[2+\frac{\gamma_{\mathrm{opt}}}{\gamma_{m}}\right]\left(\frac{\alpha_{L}-\alpha_{R}}{2}\right)\right\}, \\
& \left.\bar{S}_{I I, \text { tot }}[\omega]\right|_{\Delta=-\omega_{m}}=\bar{S}_{0}+\frac{\kappa_{R}}{\kappa} \frac{\gamma_{m} \gamma_{\mathrm{opt}}}{\left(\omega+\omega_{m}\right)^{2}+\frac{\gamma_{\mathrm{ot}}^{2}}{4}}\left\{n_{m}^{\mathrm{th}}+n_{\mathrm{eff}}^{\mathrm{th}}+\frac{\beta+\alpha_{R}}{2}-\frac{\gamma_{\mathrm{opt}}}{\gamma_{m}}\left[n_{c}^{\mathrm{th}}-n_{R}^{\mathrm{th}}\right]+\frac{\kappa_{\mathrm{L}}}{\kappa}\left[2-\frac{\gamma_{\mathrm{opt}}}{\gamma_{m}}\right]\left(\frac{\alpha_{L}-\alpha_{R}}{2}\right)\right\},
\end{aligned}
$$

where we define $n_{\mathrm{eff}}^{\text {th }}=2 n_{c}^{\text {th }}-n_{R}^{\text {th }}, \gamma_{\mathrm{tot}}=\gamma_{m} \pm \gamma_{\mathrm{opt}}$, and the noise floor

$$
\bar{S}_{0}=\frac{\alpha_{R}}{2}+n_{R}^{\mathrm{th}}+\frac{4 \kappa_{R}}{\kappa}\left(n_{c}^{\mathrm{th}}-n_{R}^{\mathrm{th}}\right)+\frac{2 \kappa_{R}}{\kappa}\left(\alpha_{L}-\alpha_{R}\right) .
$$

For the normal-ordered spectra, we obtain

$$
\begin{aligned}
& \left.S_{I I, \text { tot }}^{\mathrm{N}}[\omega]\right|_{\Delta=+\omega_{m}}=\bar{S}_{0}-\frac{\alpha_{R}}{2}+\frac{\kappa_{R}}{\kappa} \frac{\gamma_{m} \gamma_{\mathrm{opt}}}{\left(\omega-\omega_{m}\right)^{2}+\frac{\gamma_{\mathrm{tot}}^{2}}{4}}\left[n_{m}^{\mathrm{th}}-n_{\mathrm{eff}}^{\mathrm{th}}-\frac{\gamma_{\mathrm{opt}}}{\gamma_{m}}\left[n_{c}^{\mathrm{th}}-n_{R}^{\mathrm{th}}\right]\right], \\
& \left.S_{I I, \mathrm{tot}}^{\mathrm{N}}[\omega]\right|_{\Delta=-\omega_{m}}=\bar{S}_{0}-\frac{\alpha_{R}}{2}+\frac{\kappa_{R}}{\kappa} \frac{\gamma_{m} \gamma_{\mathrm{opt}}}{\left(\omega+\omega_{m}\right)^{2}+\frac{\gamma_{\mathrm{tot}}^{2}}{4}}\left[n_{m}^{\mathrm{th}}+n_{\mathrm{eff}}^{\mathrm{th}}+\beta-\frac{\gamma_{\mathrm{opt}}}{\gamma_{m}}\left[n_{c}^{\mathrm{th}}-n_{R}^{\mathrm{th}}\right]\right] .
\end{aligned}
$$


If one assumes the case of a weak optomechanical cooperativity, $\gamma_{\text {tot }} \simeq \gamma_{m}$, takes $\alpha_{L}=\alpha_{R}$, and transforms back into the lab frame, we recover the spectral densities given in the main text; see Eqs. (3), (4), (6), and (7).

It is also useful to characterize the asymmetry of the $\Delta= \pm \omega_{m}$ spectra in terms of the total integrated weight of the mechanical feature. Defining $\delta \mathcal{I}=\int(d \omega / 2 \pi) \delta S[\omega]$ and taking $\gamma_{\text {opt }} \ll \gamma_{m}$, we find

$$
\begin{aligned}
& \delta \overline{\mathcal{I}}_{I I, \text { tot }}=\frac{\kappa_{R}}{\kappa} \gamma_{\mathrm{opt}}\left[2 n_{\mathrm{eff}}^{\mathrm{th}}+\frac{\kappa_{R}}{\kappa} \alpha_{R}+\frac{\kappa_{L}}{\kappa} \alpha_{L}\right], \\
& \delta \mathcal{I}_{I I, \mathrm{tot}}^{\mathrm{N}}=\frac{\kappa_{R}}{\kappa} \gamma_{\mathrm{opt}}\left[2 n_{\mathrm{eff}}^{\mathrm{th}}+\beta\right] .
\end{aligned}
$$

Besides classical noise squashing, we thus see that the asymmetry of the symmetrized spectra (corresponding to the linear field measurement) are most naturally interpreted as being due to the contribution of fluctuations of the incident microwave fields, whereas the asymmetry in the normal-ordered spectra is most naturally attributed to the fluctuations of the mechanical oscillator.

Finally, note that the output spectra are linked via the commutation relation of the output field, which must be the same as those of the corresponding input field:

$$
\left[\hat{d}_{R, \text { out }}(\omega), \hat{d}_{R, \text { out }}^{\dagger}(\Omega)\right]=\alpha_{R} \delta(\omega+\Omega) .
$$

Calculating the commutator using the scattering matrix in Eq. (A5) and keeping $\alpha_{L}, \alpha_{R}$, and $\beta$ unspecified, we obtain for both detuning cases

$$
\begin{aligned}
{\left.\left[\hat{d}_{R, \text { out }}(\omega), \hat{d}_{R, \text { out }}^{\dagger}(\Omega)\right]\right|_{\Delta= \pm \omega_{m}}=} & \delta(\omega+\Omega)\left\{\alpha_{R}+\frac{4 \kappa_{R} \kappa_{L}}{\kappa^{2}}\left(\alpha_{L}-\alpha_{R}\right)\right. \\
& \left. \pm \frac{\kappa_{R}}{\kappa} \frac{\gamma_{m} \gamma_{\mathrm{opt}}}{\left(\omega \mp \omega_{m}\right)^{2}+\frac{\gamma_{\mathrm{ot}}^{2}}{4}}\left[\left(\beta-\frac{\kappa_{L}}{\kappa} \alpha_{L}-\frac{\kappa_{R}}{\kappa} \alpha_{R}\right) \mp \frac{\kappa_{L}}{\kappa}\left(1 \pm \frac{\gamma_{\mathrm{opt}}}{\gamma_{m}}\right)\left(\alpha_{R}-\alpha_{L}\right)\right]\right\} .
\end{aligned}
$$

We thus see that preserving the commutation relation of the output $R$ fields requires, in general, $\alpha_{L}=\alpha_{R}=\beta$. The fact that the commutator of the output field is a constant means that for any detuning, the symmetrized spectrum will be equal to the normal-ordered spectrum plus a frequency-independent noise background.

\section{Two balanced detuned tones with cooling}

In our actual experiment, we have a two-port electromechanical system that we pump during our measurement simultaneously with three microwave tones. These tones are all detuned from the cavity resonance, and in a frame rotating at the cavity frequency $\omega_{c}$, the drive Hamiltonian reads

$$
\begin{aligned}
\hat{\mathcal{H}}_{\text {drive }}= & \sum_{\nu=\mp} a_{\nu}\left(\hat{a} e^{i \nu\left(\omega_{m}+\delta\right) t}+\hat{a}^{\dagger} e^{-i \nu\left(\omega_{m}+\delta\right) t}\right) \\
& +a_{\text {cool }}\left(\hat{a} e^{-i\left(\omega_{m}+\delta_{c}\right) t}+\hat{a}^{\dagger} e^{i\left(\omega_{m}+\delta_{c}\right) t}\right) .
\end{aligned}
$$

The first term describes the two balanced detuned tones: one is in the amount of $\delta$ detuned below the red sideband $(\nu=-)$, and the other one is with the same amount detuned above the blue sideband $(\nu=+)$. The second term corresponds to the cooling tone, which we assume to be sufficiently detuned below the red sideband $\left(\delta_{c}>\delta \gg \gamma_{m}\right)$, so that the cooling tone acts independently from the probe tones; see the next section for further discussions.
Now, we start with the driving scheme in Eq. (A17) and the standard Hamiltonian in Eq. (A1), which we rotate in a frame at the cavity frequency and the mechanical frequency $\omega_{m}$. Additionally, we perform a rotating-wave approximation as usual, where we neglect nonresonant processes $\left(\omega_{m} \gg \kappa\right)$. As before, we use input-output theory to include the dissipative environment and derive the quantum Langevin equations for the fluctuation operators of the microwave (mechanical) $\hat{d}(\hat{c})$ system. By solving these Langevin equations for the noise operator $\hat{c}[\omega]$ of the mechanical oscillator, we can derive the symmetrized noise spectral density of the mechanical motion $\left(\hat{x}=\left[\hat{c}+\hat{c}^{\dagger}\right] x_{\mathrm{ZP}}\right)$ :

$$
\begin{aligned}
\bar{S}_{x x}[\omega] & =\frac{1}{2} \int d t e^{i \omega t}\langle\{\hat{x}(t), \hat{x}(0)\}\rangle \\
& =\frac{\gamma_{M}}{\omega^{2}+\frac{\gamma_{\text {tot }}^{2}}{4}}\left[\left(n_{M}^{\text {th }}+\frac{1}{2}\right)+\frac{\gamma_{\mathrm{opt}}^{-}+\gamma_{\mathrm{opt}}^{+}}{\gamma_{M}}\left(n_{c}^{\mathrm{th}}+\frac{1}{2}\right)\right] x_{\mathrm{ZP}}^{2},
\end{aligned}
$$

with the total damping $\gamma_{\text {tot }}=\gamma_{M}+\gamma_{\text {opt }}^{+}-\gamma_{\text {opt }}^{-}$, where the $\gamma_{\text {opt }}^{ \pm}=4 G_{ \pm}^{2} / \kappa$ corresponds to the optical (anti-)damping induced by the red(blue) tone. The optical damping $\gamma_{\mathrm{opt}}^{\text {cool }}$, associated with the cooling tone at $\omega=\omega_{c}-\omega_{m}-\delta_{c}$, is included in the enhanced mechanical linewidth $\gamma_{M}=\gamma_{m}+\gamma_{\mathrm{opt}}^{\mathrm{cool}}$, as well as in the modified mechanical occupation $n_{M}^{\text {th }}=\left(\gamma_{m} n_{m}^{\text {th }}+\gamma_{\text {opt }}^{\text {cool }} n_{c}^{\text {th }}\right) / \gamma_{M}$.

In the calculation of the output spectra, we assume that the anti-Stokes sideband created by the red tone and the 
Stokes sideband created by the blue tone can be treated independently. The distance between the two sidebands in frequency space is $2 \delta$; thus, for $\delta \gg \gamma_{\text {tot }}$, we have two wellseparated Lorentzians and we can neglect a direct coupling of the drives in the Langevin equations; see the next section for further discussions. The noise in the output field near the Stokes sideband $(\omega=\omega+\delta)$ and the anti-Stokes sideband $(\omega=\omega-\delta)$ becomes

$$
\begin{aligned}
\hat{d}_{R, \text { out }}[\omega \mp \delta]= & {\left[1-\frac{2 \kappa_{R}}{\kappa} \pm \frac{\kappa_{R}}{\kappa} \frac{\gamma_{\mathrm{opt}}^{ \pm}}{-i \omega+\frac{\gamma_{\mathrm{ot}}}{2}}\right] \hat{d}_{R, \text { in }}[\omega \mp \delta]-\left[2 \mp \frac{\gamma_{\mathrm{opt}}^{ \pm}}{-i \omega+\frac{\gamma_{\mathrm{tot}}}{2}}\right] \sum_{\bar{\sigma} \in L, I} \frac{\sqrt{\kappa_{R} \kappa_{\bar{\sigma}}}}{\kappa} \hat{d}_{\bar{\sigma}, \text { in }}[\omega \mp \delta] } \\
& +i \sqrt{\frac{\kappa_{\mathrm{R}}}{\kappa}} \frac{\sqrt{\gamma_{M} \gamma_{\mathrm{opt}}^{ \pm}}}{-i \omega+\frac{\gamma_{\text {tot }}}{2}} \hat{c}_{\mathrm{in}, \mathrm{tot}}^{(\dagger)}[\omega] \pm \frac{\sqrt{\gamma_{\mathrm{opt}}^{-} \gamma_{\mathrm{opt}}^{+}}}{-i \omega+\frac{\gamma_{\mathrm{tot}}}{2}} \sum_{\sigma \in L, I, R} \frac{\sqrt{\kappa_{R} \kappa_{\sigma}}}{\kappa} \hat{d}_{\sigma, \mathrm{in}}^{\dagger}[\omega \mp \delta],
\end{aligned}
$$

with the effective mechanical input noise

$$
\begin{aligned}
\hat{c}_{\mathrm{in}, \mathrm{tot}}^{(\dagger)}[\omega] & =\sqrt{\frac{\gamma_{m}}{\gamma_{M}}} \hat{c}_{\mathrm{in}}^{(\dagger)}[\omega] \\
& \mp i \sqrt{\frac{\gamma_{\mathrm{opt}}^{\mathrm{cool}}}{\gamma_{M}}} \sum_{\sigma \in L, I, R} \sqrt{\frac{\kappa_{\sigma}}{\kappa}} \hat{d}_{\sigma, \mathrm{in}}^{(\dagger)}\left[\omega \mp \delta_{c}\right],
\end{aligned}
$$

where we again use the input-output relation $\hat{d}_{R \text {,out }}=$ $\hat{d}_{R, \text { in }}+\sqrt{\kappa_{R}} \hat{d}$ and approximate the susceptibility of the cavity as $\chi_{c}[\omega]=\left[-i\left(\omega \mp \delta_{(c)}\right)+\kappa / 2\right]^{-1} \simeq 2 / \kappa$. The structure of the output-noise operator is similar to the single-tone setup; see the first row in Eq. (A5), although, here, we have also a contribution from the cooling tone and a coupling to $\hat{d}_{\sigma \text {,in }}^{\dagger}$, arising from the fact that the mechanical oscillator sees both drives and thus mediates an indirect coupling between the two sidebands. With the noise correlators and commutation relations given in Eq. (A3) and setting $\alpha_{\sigma}=\beta=1$, the symmetrized noise spectral densities are

$$
\begin{gathered}
\bar{S}_{I I, \text { tot }}[\omega-\delta]=\bar{S}_{0}+\frac{\kappa_{R}}{\kappa} \frac{\gamma_{\mathrm{tot}} \gamma_{\mathrm{opt}}^{+}}{\omega^{2}+\frac{\gamma_{\mathrm{ot}}^{2}}{4}}\left[\bar{n}_{m}-n_{\mathrm{eff}}^{\mathrm{th}}\right], \\
\bar{S}_{I I, \mathrm{tot}}[\omega+\delta]=\bar{S}_{0}+\frac{\kappa_{R}}{\kappa} \frac{\gamma_{\mathrm{tot}} \gamma_{\mathrm{opt}}^{-}}{\omega^{2}+\frac{\gamma_{\mathrm{tot}}^{2}}{4}}\left[\bar{n}_{m}+n_{\mathrm{eff}}^{\mathrm{th}}+1\right],
\end{gathered}
$$

with the averaged mechanical occupation

$$
\bar{n}_{m}=\frac{\gamma_{M}}{\gamma_{\mathrm{tot}}} n_{M}^{\mathrm{th}}+\frac{\gamma_{\mathrm{opt}}^{-}}{\gamma_{\mathrm{tot}}}\left(n_{c}^{\mathrm{th}}+1\right)+\frac{\gamma_{\mathrm{opt}}^{+}}{\gamma_{\mathrm{tot}}} n_{c}^{\mathrm{th}}
$$

and the noise floor $\bar{S}_{0}$ as defined in Eq. (A12). The normal-ordered noise spectral densities yield

$$
S_{I I, \text { tot }}^{\mathrm{N}}[\omega-\delta]=\bar{S}_{0}-\frac{1}{2}+\frac{\kappa_{R}}{\kappa} \frac{\gamma_{\mathrm{tot}} \gamma_{\mathrm{opt}}^{+}}{\omega^{2}+\frac{\gamma_{\mathrm{tot}}^{2}}{4}}\left[\bar{n}_{m}-n_{\mathrm{eff}}^{\mathrm{th}}\right],
$$

$$
\begin{aligned}
S_{I I, \mathrm{tot}}^{\mathrm{N}}[\omega+\delta]= & \bar{S}_{0}-\frac{1}{2} \\
& +\frac{\kappa_{R}}{\kappa} \frac{\gamma_{\mathrm{tot}} \gamma_{\mathrm{opt}}^{-}}{\omega^{2}+\frac{\gamma_{\mathrm{tot}}^{2}}{4}}\left[\bar{n}_{m}+n_{\mathrm{eff}}^{\mathrm{th}}+\frac{\gamma_{M}}{\gamma_{\mathrm{tot}}}+\frac{\gamma_{\mathrm{opt}}^{+}-\gamma_{\mathrm{opt}}^{-}}{\gamma_{\mathrm{tot}}}\right] .
\end{aligned}
$$

For equal coupling strengths $G_{+}=G_{-}$, the amount of optical damping and antidamping is the same, i.e., $\gamma_{\mathrm{opt}}^{+}=\gamma_{\mathrm{opt}}^{-}=\gamma_{\mathrm{opt}}$, and thus, the total damping contains only the enhanced mechanical linewidth $\gamma_{\text {tot }}=\gamma_{M}$. For the case $G_{+} \neq G_{-}$, the asymmetries in terms of their integrated weights become

$$
\begin{aligned}
\delta \overline{\mathcal{I}}_{I I, \text { tot }} & =\delta \mathcal{I}_{I I, \text { tot }}^{\mathrm{N}} \\
& =\frac{\kappa_{R}}{\kappa}\left\{\bar{n}_{m}\left[\gamma_{\mathrm{opt}}^{-}-\gamma_{\mathrm{opt}}^{+}\right]+\left(n_{\mathrm{eff}}^{\mathrm{th}}+1\right) \gamma_{\mathrm{opt}}^{-}+n_{\mathrm{eff}}^{\text {th }} \gamma_{\mathrm{opt}}^{+}\right\} .
\end{aligned}
$$

Thus, the observed sideband asymmetry of the symmetrized spectrum and the sideband asymmetry for the normal-ordered spectrum coincide. For balanced optical damping rates $\gamma_{\mathrm{opt}}^{+}=\gamma_{\mathrm{opt}}^{-}$, we obtain the expected result for the asymmetry, which scales with $2 n_{\mathrm{eff}}^{\text {th }}+1$.

\section{Effects of asymmetric parameters, the cooling tone, and the next-sideband contributions}

In our main analysis, we assumed that the direct coupling between the cavity fields at the two mechanically generated sidebands near the cavity resonance is negligible. By this, we mean that the Lorentzian-shaped resonance around $\omega_{c}-\delta$ (lab frame), created due to the drive on the red sideband, does not overlap with the one created from the drive on the blue sideband at frequency $\omega_{c}+\delta$. Within this approximation, we could derive two independent noise spectral densities, one valid for frequencies close to the Stokes sideband [Eq. (A21b)] and the other for frequencies close to the anti-Stokes sideband [Eq. (A21a)]. From these expressions, it 
follows that the width of each Lorentzian is given by the total damping rate $\gamma_{\text {tot }}$. Thus, the detuning of the control lasers from the sideband frequencies should fulfill the condition $\delta \gg \gamma_{\text {tot }}$. Briefly, we want to confirm the validity of this condition by calculating the complete RWA solution. In this case, the noise in the output field near the cavity resonance becomes $\left(G_{-}=G_{+}\right.$and rotating frame)

$$
\begin{aligned}
\hat{d}_{R, \text { out }}[\omega]= & \hat{d}_{R, \mathrm{in}}[\omega]-\sqrt{\kappa_{R}} \chi_{c}[\omega] \sum_{\sigma \in R, L, I} \sqrt{\kappa_{\sigma}} \hat{d}_{\sigma, \mathrm{in}}[\omega]-\sqrt{\frac{\kappa_{R}}{\kappa}} \gamma_{\mathrm{opt}}\left(\chi_{m}[\omega-\delta]-\chi_{m}[\omega+\delta]\right) \sum_{\sigma \in R, L, I} \sqrt{\frac{\kappa_{\sigma}}{\kappa}}\left(\hat{d}_{\sigma, \mathrm{in}}[\omega]+\hat{d}_{\sigma, \mathrm{in}}^{\dagger}[\omega]\right) \\
& +i \sqrt{\frac{\kappa_{R}}{\kappa} \gamma_{M} \gamma_{\mathrm{opt}}}\left(\chi_{m}[\omega+\delta] \hat{c}_{\mathrm{in}, \mathrm{tot}}[\omega+\delta]+\chi_{m}[\omega-\delta] \hat{c}_{\mathrm{in}, \mathrm{tot}}^{\dagger}[\omega-\delta]\right)
\end{aligned}
$$

with the susceptibilities for the mechanical oscillator $\chi_{m}[\omega]=\left[-i \omega+\left(\gamma_{M} / 2\right)\right]^{-1}$ and the microwave cavity $\chi_{c}[\omega]=[-i \omega+(\kappa / 2)]^{-1}$. Note that near the Stokes and anti-Stokes sideband, i.e., for frequencies around $\omega \sim \pm \delta$, the mechanical susceptibility shows up as $\chi_{m}[\omega]$ and $\chi_{m}[\omega \pm 2 \delta] ; \quad$ by neglecting the contribution from $\chi_{m}[\omega \pm 2 \delta]$, we recover Eq. (A19), describing two independent resonances at $\omega \sim \pm \delta$.

For simplicity, we focus on the symmetrized noise spectral density, which in this case yields

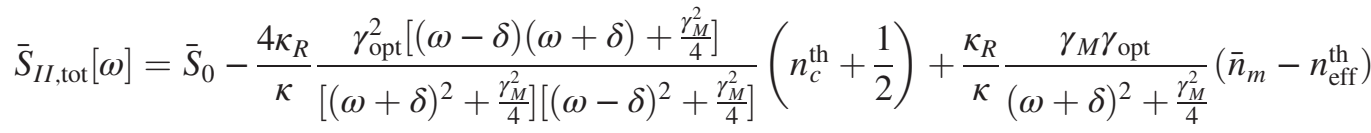

$$
\begin{aligned}
& +\frac{\kappa_{R}}{\kappa} \frac{\gamma_{M} \gamma_{\mathrm{opt}}}{(\omega-\delta)^{2}+\frac{\gamma_{M}^{2}}{4}}\left(\bar{n}_{m}+n_{\mathrm{eff}}^{\mathrm{th}}+1\right) \\
& =\bar{S}_{0}+\bar{S}_{I I, \text { tot }}^{\mathrm{mix}}[\omega]+\bar{S}_{I I, \text { tot }}^{\mathrm{AS}}[\omega]+\bar{S}_{I I, \mathrm{tot}}^{\mathrm{S}}[\omega] \text {. }
\end{aligned}
$$

Here, we have written the noise spectral density in a frame rotating at the cavity resonance frequency. This expression contains both Lorentzians near the anti-Stokes (AS) and Stokes (S) sidebands, as well as the noise floor $\bar{S}_{0}$ and a mixing term $\bar{S}_{I I, \text { tot }}^{\text {mix }}[\omega]$. Figure 4(a) shows a plot of this output spectrum for the parameters used in the experiment. Both resonances are clearly separated and each well described by the spectra calculated without a coupling of the fields (dashed red and dotted blue lines). By decreasing the detuning $\delta$, the distance between the peaks decreases and they start to overlap. Without any detuning, we end up with a Lorentzian at the cavity resonance, with an integrated weight containing solely the mechanical bath.

To study the influence of the detunings, we compare the symmetrized output spectrum [Eq. (A26)] to the single Lorentzian approximations $\bar{S}_{I I, \text { tot }}^{\mathrm{S}(\mathrm{AS})}[\omega]$. For simplicity, we focus on the maxima at the Stokes and anti-Stokes sidebands and subtract the noise floor. We end up with the ratios

$$
\frac{\bar{S}_{I I, \mathrm{tot}}[ \pm \delta]-\bar{S}_{0}}{\bar{S}_{I I, \mathrm{tot}}^{\mathrm{S}(\mathrm{AS})}[ \pm \delta]}=1+\frac{1}{\left[\frac{4 \delta}{\gamma_{M}}\right]^{2}+1}\left(\frac{n_{M}^{\mathrm{th}}-n_{\mathrm{opt}}^{\mathrm{th}}+\frac{1 \mp 1}{2}}{n_{M}^{\mathrm{th}}+n_{\mathrm{opt}}^{\mathrm{th}}+\frac{1 \pm 1}{2}}\right),
$$

with $n_{\mathrm{opt}}^{\text {th }}=\frac{\gamma_{\mathrm{opt}}}{\gamma_{M}}\left(2 n_{c}^{\text {th }}+1\right) \pm n_{\mathrm{eff}}^{\text {th }}$. From this expression, we see that the corrections to $\bar{S}_{I I, \text { tot }}^{\mathrm{S}(\mathrm{AS})}[\omega]$ (second term in the equation above) vanish as expected, if $\delta \gg \gamma_{M}$; see Fig. 4(b). Hence, in this regime, we can describe our spectra as two individual resonances. Note that the actual chosen detuning in the experiment lays clearly in the regime where both peaks are well separated; see Figs. 4(a) and 4(b).

Moreover, we want to discuss briefly the influence of the cooling tone. So far, we have worked with an effective mechanical input noise [see Eq. (A20)], where only the mechanical system is directly coupled and affected by the cooling tone. This has led to a reduced mechanical occupation $n_{M}^{\text {th }}$ and an increased mechanical linewidth $\gamma_{M}$, which we use to derive the output spectra close to the Stokes and anti-Stokes sidebands. Thus, we treat the cooling tone as part of the mechanical bath without any direct effect on the noise spectrum.

In practice, Raman processes associated with the cooling tone, although very nonresonant, could also contribute directly to the measured spectrum. These contributions are not simply additive, and to verify that they do not affect the sideband asymmetry in our experiment, we extend our theoretical description and include additional processes due to the cooling tone. We start from the time-dependent, and therewith not exactly solvable, interaction Hamiltonian 

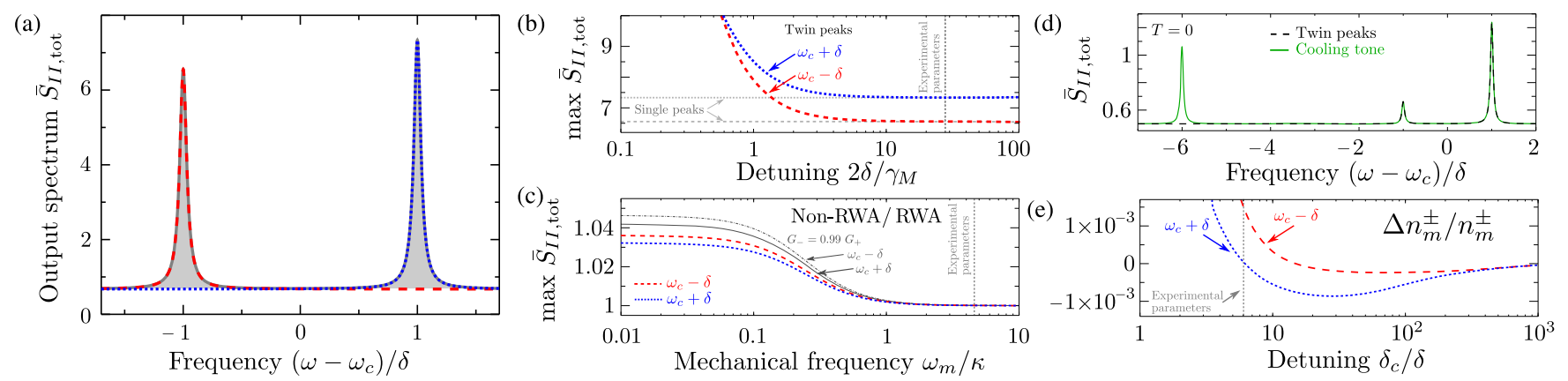

FIG. 4. Effects of asymmetric parameters, the cooling tone, and the next-sideband contributions. The dotted blue (dashed red) lines correspond to the results for the (anti-)Stokes sideband at $\omega-\omega_{c}=+\delta(-\delta)$. (a) Symmetrized output spectra as a function of frequency, for experimental parameters. The solid gray line (area) shows the non-RWA result, which exhibits two peaks at $\omega=\omega_{c} \pm \delta$. The dashed red and dotted blue lines correspond to two independent noise spectral densities, one valid for frequencies close to the anti-Stokes sideband [Eq. (A21a)] and the other one for frequencies close to the Stokes sideband [Eq. (A21b)], which each accurately describe the resonant peaks at $\omega-\omega_{c}=\mp \delta$. (b) Influence of the detuning parameter $\delta$ on the output noise. For $\delta \gg \gamma_{\text {tot }}=\gamma_{M}$, the results from the twin peak spectrum, i.e., from Eq. (A26), coincide with the single-peak approximations (dashed and dotted gray lines). (c) Effect of nextsideband contributions on the noise maximum. The gray lines include a 1\% mismatch of the coupling strengths. (d) Symmetrized output spectra for zero-temperature baths with (solid light green line) and without (dashed black line) direct contributions from the cooling tone (experimental parameters). See the text for details. (e) Influence of the detuning $\delta_{c}$ of the cooling beam on the sidebands. Plotted is $\Delta n_{m}^{ \pm} / n_{m}^{ \pm}=\left(n_{m}^{ \pm}-n_{m, 0}^{ \pm}\right) / n_{m}^{ \pm}$, where $n_{m, 0}^{ \pm}\left(n_{m}^{ \pm}\right)$corresponds to the sideband's weight without (with) direct contributions from the cooling tone. Experimental parameters: $\omega_{m} /(2 \pi)=4 \mathrm{MHz}, \gamma_{M} /(2 \pi)=360 \mathrm{~Hz}, \gamma_{m} /(2 \pi)=10 \mathrm{~Hz}, \delta /(2 \pi)=5 \mathrm{kHz}, \delta_{c} /(2 \pi)=30 \mathrm{kHz}$, $\kappa /(2 \pi)=870 \mathrm{kHz}, \kappa_{R} /(2 \pi)=450 \mathrm{kHz}, \kappa_{L} /(2 \pi)=155 \mathrm{kHz}$, and $\gamma_{\mathrm{opt}} /(2 \pi)=100 \mathrm{~Hz}$. For the cavity baths, we assume $n_{R}^{\text {th }}=n_{L}^{\text {th }}=$ 0.3 and $n_{c}^{\text {th }}=0.24$, and for the mechanical bath, we assume $n_{M}^{\text {th }}=10$.

$$
\begin{aligned}
\hat{\mathcal{H}}_{\mathrm{int}}= & \hat{d}^{\dagger} \hat{c} e^{i \delta t}\left(G+G_{c} e^{-i \Delta_{c} t}\right)+\hat{d} \hat{c}^{\dagger} e^{-i \delta t}\left(G+G_{c} e^{i \Delta_{c} t}\right) \\
& +G\left[\hat{d}^{\dagger} \hat{c}^{\dagger} e^{-i \delta t}+\hat{d} \hat{c} e^{i \delta t}\right],
\end{aligned}
$$

with the relative detuning $\Delta_{c} \equiv \delta-\delta_{c}$. Here, sidebands are created at multiples of $\Delta_{c}$, and thus, the resulting system of Langevin equations has to be truncated. In Fig. 4(d), we show results for the symmetrized output spectrum, including contributions from the next sidebands at $\pm \Delta_{c}$. The detuning of the cooling tone is chosen to be $\delta_{c}=6 \delta$, as in the experiment. The additional peak at $\omega-\omega_{c}=-\delta_{c}$ is well separated from the Stokes and anti-Stokes peaks at $\omega-\omega_{c}=\mp \delta$. Hence, a measurement of the asymmetry around these probe sidebands should not be crucially affected by the cooling peak.

To quantify this effect, we plot in Fig. 4(e) the deviations

$$
\frac{\Delta n_{m}^{ \pm}}{n_{m}^{ \pm}}=\frac{n_{m}^{ \pm}-n_{m, 0}^{ \pm}}{n_{m}^{ \pm}},
$$

where $n_{m}^{ \pm}\left(n_{m, 0}^{ \pm}\right)$corresponds to the areas of the sidebands with (without) the additional contributions from the cooling tone. The numerical results show that the deviations are small for appropriate detuning $\delta_{c}$; e.g., for the experimental parameter set, they are in the order of $\Delta n_{m}^{+} / n_{m}^{+} \sim 10^{-3}$ and $\Delta n_{m}^{-} / n_{m}^{-} \sim 10^{-5}$. In comparison to the asymmetry of the sidebands, which can be estimated to be at the order of $2 n_{\mathrm{eff}}^{\text {th }}+1$, these deviations are negligible.

Finally, we want to briefly comment on the influence of higher-order mechanical sidebands. The general linearized interaction Hamiltonian for our setup reads

$$
\begin{aligned}
\hat{\mathcal{H}}_{\mathrm{int}}= & G_{+}\left[e^{-i \delta t} \hat{d} \hat{c}^{\dagger}+e^{i \delta t} \hat{d}^{\dagger} \hat{c}\right]+G_{-}\left[e^{i \delta t} \hat{d} \hat{c}+e^{-i \delta t} \hat{d}^{\dagger} \hat{c}^{\dagger}\right] \\
& +\hat{\mathcal{H}}_{\mathrm{CR}}, \\
\hat{\mathcal{H}}_{\mathrm{CR}}= & G_{+}\left[e^{i\left(2 \omega_{m}+\delta\right) t} \hat{d}^{\dagger} \hat{c}^{\dagger}+e^{-i\left(2 \omega_{m}+\delta\right) t} \hat{d} \hat{c}\right] \\
& +G_{-}\left[e^{i\left(2 \omega_{m}+\delta\right) t} \hat{d} \hat{c}^{\dagger}+e^{-i\left(2 \omega_{m}+\delta\right) t} \hat{d}^{\dagger} \hat{c}\right],
\end{aligned}
$$

where the counterrotating terms in $\hat{\mathcal{H}}_{\mathrm{CR}}$ describe the strongly nonresonant Stokes and anti-Stokes processes generated by the two control lasers. The coupling strengths $G_{ \pm}$contain the drive amplitudes, as usual, but we assume again that they can be different in magnitude, which leads to a total mechanical damping of $\gamma_{\mathrm{opt}}=\gamma_{M}+\gamma_{\mathrm{opt}}^{+}-\gamma_{\mathrm{opt}}^{-}$ with $\gamma_{\mathrm{opt}}^{ \pm}=4 G_{ \pm} / \kappa$. Note that for the system to be stable, the total damping has to be positive, which roughly translates into the condition $G_{+}>G_{-}$.

The inclusion of the counterrotating terms leads to a time-dependent problem, which cannot be solved exactly. In principle, $\hat{\mathcal{H}}_{\mathrm{CR}}$ generates an infinite number of sidebands at multiples from $\pm \omega_{m}$. If one is not too far from the resolved-sideband limit, a perturbative approach keeping track of only the leading-order sidebands created by $\hat{\mathcal{H}}_{\mathrm{CR}}$ is sufficient. Figure 4(c) depicts the deviations of the maximum of the symmetrized spectral density as a function of $\omega_{m} / \kappa$, including the next sidebands at frequencies $\omega=\omega_{c} \pm 2 \omega_{m}$. As expected, when one even modestly approaches the resolved-sideband regime, i.e., $\omega_{m}>\kappa$, the contributions from the counterrotating terms are negligible. Moreover, for the given experimental setup, we are far in the resolved-sideband regime, as indicated in the graph. 


\section{APPENDIX B: LINEAR-RESPONSE THEORY}

In this Appendix, we briefly review the linear response approach to understand the sideband asymmetry observed using linear field detection; this explanation was first discussed in Ref. [16]. For linear field detection, the observed asymmetry can be fully attributed to noise correlations in the detector (in this case, the driven cavity), correlations that could exist classically. We generalize the discussion of Ref. [16] to include thermal noise driving the cavity, showing that the same backaction-imprecision correlations allow one to understand the squashing of thermal noise seen in previous experiments [4]. We also show that the particular value of the backaction-imprecision correlator, required to account for the zero-temperature sideband asymmetry, plays a special role in the linearresponse approach to quantum measurements $[7,28]$ : it is precisely the value needed to ensure there is no additional constraint on the detector's symmetrized noise correlators besides what would exist classically.

Following Ref. [7], the general linear response approach starts by assuming a linear coupling between the detector and the observable to be measured (in this case, $\hat{x}$, the mechanical position):

$$
\hat{\mathcal{H}}_{\text {int }}=-A \hat{x} \hat{F} .
$$

Here, $\hat{F}$ is the detector quantity that couples to the measured system and plays the role of a backaction force. In our optomechanical case, we have $\hat{F}=-\left(g / x_{\mathrm{ZP}}\right) \hat{a}^{\dagger} \hat{a}$. $A$ is a dimensionless coupling constant that we will use to track the order at which $\hat{H}_{\text {int }}$ appears in expressions; we will set it to one at the end of the calculation.

Next, consider the detector-output observable $\hat{I}$. We assume that this quantity responds linearly to the mechanical position

$$
\langle\hat{I}[\omega]\rangle=-A \chi_{I F}[\omega]\langle\hat{x}[\omega]\rangle,
$$

where $\chi_{I F}[\omega]$ is the response coefficient or "forward gain" of the detector; it is given by a standard Kubo formula. We are interested in understanding the fluctuations of the detector output. Quantum linear response theory tells us that these fluctuations can be completely understood within an equivalent classical stochastic model $[7,28]$, where we now replace the operators $\hat{I}(t), \hat{F}(t)$, and $\hat{x}(t)$ by classical random variables. The fluctuations of the output in this model are written as

$$
\delta I_{\text {tot }}[\omega]=\delta I[\omega]-A \chi_{I F}[\omega]\left(\delta x_{0}[\omega]+A \delta x_{\mathrm{BA}}[\omega]\right) .
$$

The first term here represents the intrinsic fluctuations of the output in the absence of any coupling to the mechanics (the imprecision noise). $\delta x_{0}[\omega]$ describes the position fluctuations of the mechanics in the absence of any backaction, whereas $\delta x_{\mathrm{BA}}[\omega]$ describes the additional backaction-driven fluctuations of the mechanical resonator. $\delta x_{0}[\omega]$ is due to the intrinsic mechanical dissipation. Assuming this dissipation to be in thermal equilibrium, these fluctuations are described by the spectral density

$$
\begin{aligned}
\mathcal{S}_{x x, 0}[\omega] \equiv\left\langle\left|\delta x_{0}[\omega]\right|^{2}\right\rangle & =-\hbar \operatorname{Im} \chi_{x x}[\omega] \operatorname{coth} \beta \hbar \omega / 2 \\
& \simeq-\hbar \operatorname{Im} \chi_{x x}[\omega]\left(1+2 n_{m}^{\mathrm{th}}\right),
\end{aligned}
$$

where $\chi_{x x}[\omega]$ is the mechanical force susceptibility

$$
\chi_{x x}(\omega)=\frac{1 / m}{\left(\omega^{2}-\omega_{m}^{2}\right)+i \omega \gamma_{m}} .
$$

Similarly, the backaction-driven position fluctuations are described by

$$
\mathcal{S}_{x x, \mathrm{BA}}[\omega] \equiv\left\langle\left|\delta x_{\mathrm{BA}}[\omega]\right|^{2}\right\rangle=\left|\chi_{x x}[\omega]\right|^{2} \mathcal{S}_{F F}[\omega] .
$$

The spectral densities of the output fluctuations are then given by

$$
\begin{aligned}
\mathcal{S}_{I I, \mathrm{tot}}[\omega]= & \mathcal{S}_{I I}[\omega]+A^{2}\left|\chi_{I F}[\omega]\right|^{2}\left[\mathcal{S}_{x x, 0}[\omega]+A^{2} \mathcal{S}_{x x, \mathrm{BA}}[\omega]\right. \\
& \left.-2 \operatorname{Re}\left(\chi_{x x}[\omega]^{*} \frac{\mathcal{S}_{I F}[\omega]}{\chi_{I F}[\omega]}\right)\right] \\
= & \mathcal{S}_{I I}[\omega]+\left|\chi_{I F}[\omega]\right|^{2}\left(S_{x x, \mathrm{eff}}[\omega]\right)
\end{aligned}
$$

Here, $\mathcal{S}_{I F}[\omega]$ is the spectral density that describes any possible correlations between the intrinsic imprecisionnoise contribution to the output $(\delta I[\omega])$ and the backaction-force noise driving the mechanics $(\delta F[\omega])$. In the quantum theory, an identical expression to the above holds, except all classical noise spectral densities $\mathcal{S}_{A B}[\omega]$ describing the detector, are replaced by the corresponding symmetrized quantum noise spectral densities $\bar{S}_{A B}[\omega][7]:$

$$
\bar{S}_{A B}[\omega]=\frac{1}{2} \int_{-\infty}^{\infty} d t\langle\{\hat{A}(t), \hat{B}(0)\}\rangle .
$$

For our optomechanical system, the needed detectorcorrelation functions are easily computed from the linearized Heisenberg-Langevin equations. As in the main text, we assume a two-sided cavity and measure the quantity $\hat{I}$ defined below Eq. (A7). One finds

$$
\begin{aligned}
\chi_{I F}[\omega] & =-i \frac{\sqrt{\kappa_{R}} G}{x_{\mathrm{ZP}}}\left(\chi_{c}[\omega]-\chi_{c}[-\omega]^{*}\right), \\
\bar{S}_{I I}[\omega]= & \sum_{\nu= \pm}\left\{\left|1-\kappa_{R} \chi_{c}[\nu \omega]\right|^{2}\left[(1 / 2)+n_{R}^{\mathrm{th}}\right]\right. \\
& \left.+\kappa_{R} \kappa_{L}\left|\chi_{c}[\nu \omega]\right|^{2}\left[(1 / 2)+n_{L}^{\mathrm{th}}\right]\right\},
\end{aligned}
$$




$$
\begin{aligned}
& \bar{S}_{F F}[\omega]=\frac{G^{2}}{x_{\mathrm{ZP}}^{2}}\left(\left|\chi_{c}[\omega]\right|^{2}+\left|\chi_{c}[-\omega]\right|^{2}\right) \sum_{\sigma=L, R} \kappa_{\sigma}\left[(1 / 2)+n_{\sigma}^{\mathrm{th}}\right], \\
& \bar{S}_{I F}[\omega]=-\frac{\sqrt{\kappa_{R}} G}{x_{\mathrm{ZP}}} \sum_{\sigma=L, R}\left(\Lambda_{\sigma}[\omega]+\Lambda_{\sigma}[-\omega]^{*}\right)\left[(1 / 2)+n_{\sigma}^{\mathrm{th}}\right],
\end{aligned}
$$

where for the cross-correlator, we have introduced the functions

$$
\begin{aligned}
& \Lambda_{R}[\omega]=-\left(1-\kappa_{R} \chi_{c}[\omega]\right) \chi_{c}[\omega]^{*}, \\
& \Lambda_{L}[\omega]=-\kappa_{L}\left|\chi_{c}[\omega]\right|^{2} .
\end{aligned}
$$

We are again interested in the symmetrized output spectrum of the detector in a narrow range (in the order of $\gamma_{m}$ ) near the cavity resonance frequency, for a drive detuning $\Delta= \pm \omega_{m}$; as always, we consider the goodcavity limit $\omega_{m} \gg \kappa$. Over this range of frequencies, we can neglect the frequency dependence of the cavity correlators and evaluate them on resonance (i.e., $\omega=\Delta$ in the rotating frame). Of particular interest is the cross-correlator. One finds

$$
\begin{aligned}
\bar{S}_{z F}[\Delta] \equiv & \frac{\bar{S}_{I F}[\Delta]}{\chi_{I F}[\Delta]} \\
\simeq & \mp i \hbar\left[\left(\frac{2 \kappa_{R}}{\kappa}-1\right)\left[(1 / 2)+n_{R}^{\mathrm{th}}\right]\right. \\
& \left.+\left(\frac{2 \kappa_{L}}{\kappa}\right)\left[(1 / 2)+n_{L}^{\mathrm{th}}\right]\right] \\
= & \mp i \hbar\left(\frac{1}{2}+2 n_{c}^{\mathrm{th}}-n_{R}^{\mathrm{th}}\right),
\end{aligned}
$$

where the minus sign (plus sign) corresponds to the drive detuning $\Delta=+\omega_{m}\left(\Delta=-\omega_{m}\right)$.

We see that $\bar{S}_{z F}$ is purely imaginary and changes sign for the two choices of detuning; in contrast, one can confirm that $\left|\chi_{I F}\right|, \bar{S}_{I I}$, and $\bar{S}_{F F}$ at resonance are the same for $\Delta= \pm \omega_{m}$. It immediately follows that the asymmetry between the spectra obtained at $\Delta=-\omega_{m}$ and $\Delta=\omega_{m}$ is entirely due to the detector backaction-imprecision correlations described by $\bar{S}_{z F}$.

Returning to Eq. (B7) for the output spectrum, we further note that for a sufficiently weak detector-system coupling, the term $\mathcal{S}_{x x, \mathrm{BA}}$ will be negligible to the term $\mathcal{S}_{x x, 0}$, as the backaction term is second order in the coupling (i.e., $\propto A^{2}$ ). However, the last correlation term remains significant: Its contribution relative to $\mathcal{S}_{x x, 0}$ is independent of coupling strength. In our case, where $\mathcal{S}_{I F} / \chi_{I F} \equiv \mathcal{S}_{z F}$ is purely imaginary, we can combine the leading mechanical contributions to the output spectrum as

$$
\begin{aligned}
\mathcal{S}_{x x, \mathrm{eff}}[\omega] & \simeq \mathcal{S}_{x x, 0}[\omega]-2 \operatorname{Re}\left(\chi_{x x}[\omega]^{*} \mathcal{S}_{z F}\right) \\
& =-\operatorname{Im} \chi_{x x}[\omega]\left(\hbar \operatorname{coth} \beta \hbar \omega / 2+2 \operatorname{Im} \mathcal{S}_{z F}\right) \\
& \simeq-\operatorname{Im} \chi_{x x}[\omega]\left[\hbar\left(1+2 n_{m}^{\text {th }}\right)+2 \operatorname{Im} \mathcal{S}_{z F}\right] .
\end{aligned}
$$

We see that the mechanics will give rise to a Lorentzian signature in the output spectrum but that the presence of imaginary backaction-imprecision correlations modifies the weight of the Lorentzian-it no longer simply reflects the mechanical temperature. This modified weight results in the well-known phenomenon of noise squashing.

Using Eq. (B17) for the cross-correlator, we see that this linear response calculation reproduces the asymmetry found earlier between spectra obtained for $\Delta= \pm \omega_{m}$. This approach emphasizes the fact that the asymmetry can be completely attributed to the detector, namely, the presence of backaction-imprecision correlations. These correlations are purely imaginary; the only difference between the cases is the sign of the correlator. For $\Delta=\omega_{m}$, the correlations are positive and serve to decrease the weight of the mechanical Lorentzian; they completely cancel the contribution if the mechanics is at zero temperature. For $\Delta=-\omega_{m}$, they instead serve to increase the mechanical contribution. In the absence of thermal cavity noise, the effect of the noise correlations is to cause the weight of the mechanical Lorentzian in the output spectrum to have the expected form for phonon emission or absorption: For $\Delta=\omega_{m}$, we have the emission factor $n_{m}^{\text {th }}$, and for $\Delta=-\omega_{m}$, we have $n_{m}^{\text {th }}+1$.

Besides classical noise squashing, we thus see that the asymmetry can be interpreted in terms of a finely tuned backaction-imprecision correlation. We stress that a completely classical detector could have an identical noise correlation. Nonetheless, this value of correlation plays an extremely special role in the theory of quantum limits on linear quantum detectors and amplifiers [7]. Quantum limits on such detectors (e.g., on their added noise or noise temperature) follow from a fundamental Heisenberglike inequality on their noise properties at each frequency. These inequalities take the form

$\bar{S}_{z z}[\omega] \bar{S}_{F F}[\omega]-\left|\bar{S}_{z F}[\omega]\right|^{2} \geq \frac{\hbar^{2}}{4}\left(1+\Delta\left[\frac{2 \bar{S}_{z F}[\omega]}{\hbar}\right]\right)$,

where

$$
\Delta[y]=\frac{\left|1+y^{2}\right|-\left(1+|y|^{2}\right)}{2} .
$$

We have defined $\bar{S}_{z z}[\omega]=\bar{S}_{I I}[\omega] /\left|\chi_{I F}[\omega]\right|^{2}$ and have specialized to the case relevant here, where the reverse gain of the detector vanishes. Note that for any complex number $y$, $1+\Delta[y] \geq 0$, and hence, in general, the rhs of the inequality in Eq. (B20) is nonzero. This property means that, in general, quantum mechanics makes it impossible for the 
detector backaction and imprecision noises to be perfectly correlated [i.e., the lhs of Eq. (B20) cannot, in general, be zero]. This represents a purely quantum constraint on the detector's noise properties. In the case where $\bar{S}_{z F}$ is purely real (or 0), the rhs simply reduces to $(\hbar / 2)^{2}$.

In contrast, if $\bar{S}_{z F}$ is purely imaginary, the rhs of Eq. (B20) can be reduced below $\hbar^{2} / 4$. One easily finds that it achieves the minimum value of 0 when $\bar{S}_{z F}= \pm i \hbar / 2$. Thus, for this special choice of crosscorrelation, quantum mechanics does not forbid a perfect correlation between the detector's backaction and imprecision noises. This special value is precisely what was found above for our cavity optomechanical detector at zero temperature (at the cavity resonance, with a drive detuning $\Delta= \pm \omega_{m}$, and in the good-cavity limit); see Eq. (B17). Thus, not only does this special value of cross-correlation yield an imbalance between the $\Delta= \pm \omega_{m}$ output spectra in just the way expected for quantum emission or absorption, it also implies that there is no additional Heisenberg constraint on the detector-noise properties. As discussed in Sec. IVA 4 of Ref. [7], this means that, in principle, one could make the added noise of this position detector strictly zero. As is also discussed in this reference, this vanishing does not constitute a violation of the quantum limit on position detection or amplification. One can demonstrate that in this case, the detector does not provide any amplification of the mechanical motion: the dimensionless power gain of the detector is, at most, order 1 (see Appendix I 2 of Ref. [7]). As there is no amplification, quantum mechanics does not require any added noise.

[1] V. B. Braginsky and F. Y. Khalili, Quantum Measurement (Cambridge University Press, Cambridge, England, 1992).

[2] D. H. Santamore, A. C. Doherty, and M. C. Cross, Quantum Nondemolition Measurement of Fock States of Mesoscopic Mechanical Oscillators, Phys. Rev. B 70, 144301 (2004).

[3] A. A. Clerk, F. Marquardt, and J. G. E. Harris, Quantum Measurement of Phonon Shot Noise, Phys. Rev. Lett. 104, 213603 (2010).

[4] T. Roucheleau, T. Ndukum, C. Macklin, J. B. Hertzberg, A. A. Clerk, and K. C. Schwab, Preparation and Detection of a Mechanical Resonator near the Ground State of Motion, Nature (London) 463, 72 (2010).

[5] J. D. Teufel, T. Donner, D. Li, J. W. Harlow, M. S. Allman, K. Cicak, A. J. Sirois, J. D. Whittaker, K. W. Lehnert, and R. W. Simmonds, Sideband Cooling of Micromechanical Motion to the Quantum Ground State, Nature (London) 475, 359 (2011).

[6] A. Muthukrishnan, M. O. Scully, and M. S. Zubairy, The Nature of Light: What Is a Photon? (CRC, Boca Raton, FL, 2008), p. 37-57.

[7] A. A. Clerk, M. H. Devoret, S. M. Girvin, F. Marquardt, and R. J. Schoelkopf, Introduction to Quantum Noise, Measurement, and Amplification, Rev. Mod. Phys. 82, 1155 (2010).
[8] F. Diedrich, J. C. Bergquist, W. M. Itano, and D. J. Wineland, Laser Cooling to the Zero-Point Energy of Motion, Phys. Rev. Lett. 62, 403 (1989).

[9] P. S. Jessen, C. Gerz, P. D. Lett, W. D. Phillips, S. L. Rolston, R. J. C. Spreeuw, and C. I. Westbrook, Observation of Quantized Motion of Rb Atoms in an Optical Field, Phys. Rev. Lett. 69, 49 (1992).

[10] C. Monroe, D. M. Meekhof, B. E. King, S. R. Jefferts, W. M. Itano, D. J. Wineland, and P. Gould, ResolvedSideband Raman Cooling of a Bound Atom to the 3D Zero-Point Energy, Phys. Rev. Lett. 75, 4011 (1995).

[11] J. Chan, T. P. M. Alegre, A. H. Safavi-Naeini, J. T. Hill, A. Krause, S. Gröblacher, M. Aspelmeyer, and O. Painter, Laser Cooling of a Nanomechanical Oscillator into Its Quantum Ground State, Nature (London) 478, 89 (2011).

[12] T. P. Purdy, R. W. Peterson, and C. A. Regal, Observation of Radiation Pressure Shot Noise on a Macroscopic Object, Science 339, 801 (2013).

[13] J. Suh, A. J. Weinstein, C. U. Lei, E. E. Wollman, S. K. Steinke, P. Meystre, A. A. Clerk, and K. C. Schwab, Mechanically Detecting and Avoiding the Quantum Fluctuations of a Microwave Field, Science 344, 1262 (2014).

[14] A. H. Safavi-Naeini, J. Chan, J. T. Hill, T. P. M. Alegre, A. Krause, and O. Painter, Observation of Quantum Motion of a Nanomechanical Resonator, Phys. Rev. Lett. 108, 033602 (2012).

[15] N. Brahms, T. Botter, S. Schreppler, D. W. C. Brooks, and D. M. Stamper-Kurn, Optical Detection of the Quantization of Collective Atomic Motion, Phys. Rev. Lett. 108, 133601 (2012).

[16] F. Y. Khalili, H. Miao, H. Yang, A. H. Safavi-Naeini, O. Painter, and Y. Chen, Quantum Back-action in Measurements of Zero-Point Mechanical Oscillations, Phys. Rev. A 86, 033840 (2012).

[17] D. F. Walls and G. J. Milburn, Quantum Optics (SpringerVerlag, Berlin, 1995).

[18] J. Suh, M. D. Shaw, H. G. LeDuc, A. J. Weinstein, and K. C. Schwab, Thermally Induced Parametric Instability in a Backaction Evading Measurement of a Micromechanical Quadrature near the Zero-Point Level, Nano Lett. 12, 6260 (2012).

[19] A. H. Safavi-Naeini, J. Chan, J. T. Hill, S. Gröblacher, H. Miao, Y. Chen, M. Aspelmeyer, and O. Painter, Laser Noise in Cavity-Optomechanical Cooling and Thermometry, New J. Phys. 15, 035007 (2013).

[20] J. Suh, A. J. Weinstein, and K. C. Schwab, Optomechanical Effects of Two-Level Systems in a Back-action Evading Measurement of Micro-mechanical Motion, Appl. Phys. Lett. 103, 052604 (2013).

[21] See Supplemental Material at http://link.aps.org/ supplemental/10.1103/PhysRevX.4.041003 for device calibration details.

[22] F. Marquardt, J. P. Chen, A. A. Clerk, and S. M. Girvin, Quantum Theory of Cavity-Assisted Sideband Cooling of Mechanical Motion, Phys. Rev. Lett. 99, 093902 (2007).

[23] I. Wilson-Rae, N. Nooshi, W. Zwerger, and T. J. Kippenberg, Theory of Ground State Cooling of a Mechanical Oscillator Using Dynamical Backaction, Phys. Rev. Lett. 99, 093901 (2007).

[24] J. B. Hertzberg, T. Rocheleau, T. Ndukum, M. Savva, A. A. Clerk, and K. C. Schwab, Backaction Evading Measurements of Nanomechanical Motion, Nat. Phys. 6, 213 (2010). 
[25] T. P. Purdy, P. L. Yu, N. S. Kampel, R. W. Peterson, K. Cicak, R. W. Simmonds, and C. A. Regal, Optomechanical Raman-Ratio Thermometry, arXiv:1406.7247.

[26] D. Lee, M. Underwood, D. Mason, A. B. Shkarin, K. Borkje, S. Girvin, and J.G. E. Harris, Observation of Quantum Motion in a Nanogram-Scale Object, arXiv:1406.7254.
[27] In this work, the Fourier transform is defined such that $\hat{f}(\omega)=$ $\int_{-\infty}^{\infty} d \hat{t} \hat{f}(t) e^{i \omega t}$ and $\hat{f}^{\dagger}(\omega)=\int_{-\infty}^{\infty} d t \hat{f}^{\dagger}(t) e^{i \omega t}=[\hat{f}(-\omega)]^{\dagger}$.

[28] A. A. Clerk, Quantum-Limited Position Detection and Amplification: A Linear Response Perspective, Phys. Rev. B 70, 245306 (2004). 